TRANSACTIONS OF THE

AMERICAN MATHEMATICAL SOCIETY

Volume 352, Number 3 , Pages 1217-1245

$\mathrm{S} 0002-9947(99) 02526-\mathrm{X}$

Article electronically published on September 20, 1999

\title{
SYMMETRY OF GROUND STATES FOR A SEMILINEAR ELLIPTIC SYSTEM
}

\author{
HENGHUI ZOU
}

\begin{abstract}
Let $n \geq 3$ and consider the following system
\end{abstract}

$$
\Delta \mathbf{u}+\mathbf{f}(\mathbf{u})=0, \quad \mathbf{u}>0, \quad x \in \mathbf{R}^{n} .
$$

By using the Alexandrov-Serrin moving plane method, we show that under suitable assumptions every slow decay solution of (I) must be radially symmetric.

\section{INTRODUCTION}

In an elegant paper [8], Gidas, Ni and Nirenberg considered the question of symmetry of positive $\left(C^{2}\right.$, which will be assumed throughout the paper) solutions of semilinear problems of the form

$$
\Delta u+f(u)=0, \quad x \in \mathbf{R}^{n}(n \geq 3) .
$$

Combining the well-known moving plane technique with appropriate sharp asymptotics at infinity, they showed all positive solutions of (I) with a 'fast decay' at infinity must be radially symmetric about some point in $\mathbf{R}^{n}$. In [3], Caffarelli, Gidas and Spruck introduced an alternative approach. Instead of directly developing asymptotics at infinity, they used a Kelvin transform to carry out the moving plane procedure. As a result, for certain nonlinearities with a critical or subcritical growth, they were able to obtain radial symmetry without imposing any decay assumption on solutions.

In recent papers [19]-[21], the author developed the following asymptotic expansion at infinity

$$
u(x)=\bar{u}(|x|)+\frac{\nu(|x|, \theta)}{|x|^{1+\alpha}}
$$

for 'slow decay' solutions of $(\mathrm{I})$, where $(|x|, \theta)$ are spherical coordinates, $\alpha>0$ is determined by the growth of $f$, and $\nu$ is a smooth function of its arguments having 'nice' properties near $|x|=\infty$. Using this expansion, symmetry was obtained again via moving planes.

It is observed that, for suitable nonlinearities, appropriately fast decay solutions of (I) have a removable singularity at infinity. That is, there exists a critical decay rate $\alpha>0$ only depending on the nonlinearity in the sense that if

$$
u(x)=o\left(|x|^{-\alpha}\right) \text {, }
$$

Received by the editors April 4, 1997 and, in revised form, October 20, 1997.

1991 Mathematics Subject Classification. Primary 35B40, 35J60.

Research supported in part by NSF Grants DMS-9418779 and DMS-9622937, an Alabama EPSCoR grant and a faculty research grant of the University of Alabama at Birmingham. 
then necessarily

$$
u(x)=O\left(|x|^{2-n}\right) .
$$

(Of course $\alpha<n-2$.) Positive solutions with the decay (1.2) (or equivalently (1.1) are called fast decay. Near infinity, one can expect that fast decay solutions behave very 'nicely' and therefore the procedure of moving planes can be proceeded.

In the contrary, positive solutions satisfying

$$
u(x)=O\left(|x|^{-\alpha}\right), \quad \limsup _{|x| \rightarrow \infty}|x|^{\alpha} u(x)>0
$$

are called slow decay. Slow decay solutions exist naturally, for example, for the well-known Lane-Emden equation (see [19], [21] and references therein)

$$
f(u)=u^{p}, \quad p>\frac{n+2}{n-2}
$$

and the equation (with mixed Sobolev growth)

$$
f(u)=u^{q}+u^{p}, \quad \frac{n}{n-2}<q<\frac{n+2}{n-2}<p .
$$

One can always obtain a fast decay at infinity via the Kelvin transform (see [3]), however, a subcritical or critical growth is essential to the completion of the moving plane procedure, due to a possible resulted singularity at the new origin. Indeed, in the presence of a slow decay (i.e., supercritical growth), the Kelvin transform simply inverts a non-removable singularity from infinity to the origin and is no longer useful in this case.

In this paper, we consider a prototype model of 2-systems

$$
\Delta \mathbf{u}+\mathbf{f}(\mathbf{u})=0, \quad x \in \mathbf{R}^{n}(n \geq 3),
$$

where $\mathbf{u}=\left(u_{1}, u_{2}\right)$ and

$$
\mathbf{f}(\mathbf{u})=\left(u_{2}^{p}, u_{1}^{q}\right), \quad p, q>1 .
$$

This system, referred to as the Lane-Emden system, and its parabolic analogue, referred to as the Fujita system, arise in chemical, biological and physical studies, and have been broadly investigated, see for example [5]-[7], [11], [10], [13]-[15] and references therein.

The so-called critical hyperbola

$$
\frac{1}{p+1}+\frac{1}{q+1}=\frac{n-2}{n},
$$

plays an important role in the theory of existence and non-existence for (II). Indeed, it has been shown that system (II) admits positive solutions if and only if $(p, q)$ is on or above the hyperbola, for certain important cases ([14], [15]). In particular, (II) has infinitely-many positive radial solutions when $(p, q)$ is critical, or supercritical, i.e., on or above the hyperbola (1.3); see [15]. This should not be surprising in view of the well-known fact that the Sobolev exponent $l=(n+2) /(n-2)$ divides existence and non-existence for the Lane-Emden equation.

We shall investigate the symmetry and the local behavior of positive solutions of (II). Specifically, we want to extend the results for the scalar case (cf. [19]) to the vector case (system (II)). Similarly as in the scalar case (cf. [19]), the critical hyperbola corresponding to the dimension $n-1$ given by

$$
\frac{1}{p+1}+\frac{1}{q+1}=\frac{n-3}{n-1}
$$

comes into play. 
We first introduce some notation. Put

$$
\begin{gathered}
\alpha_{1}=\frac{2(p+1)}{p q-1}>0, \quad \alpha_{2}=\frac{2(q+1)}{p q-1}, \\
\lambda_{1}=\alpha_{1}\left(n-2-\alpha_{1}\right)>0, \quad \lambda_{2}=\alpha_{2}\left(n-2-\alpha_{2}\right)>0 .
\end{gathered}
$$

and

$$
L_{1}=\left(\lambda_{1} \lambda_{2}^{p}\right)^{1 /(p q-1)}, \quad L_{2}=\left(\lambda_{1}^{q} \lambda_{2}\right)^{1 /(p q-1)} .
$$

Throughout the paper we write vector-valued functions using bold face type, e.g., $\mathbf{u}^{T}(x)=\left(u_{1}(x), u_{2}(x)\right)$, and we say that $\mathbf{u}$ is positive (non-negative) if both components are positive (non-negative).

Denoting spherical coordinates by $(r, \theta)$ with $r=|x|$, we have the following main result.

Theorem 1.1. Let $\mathbf{u}$ be a positive solution of (II). Suppose

$$
\lim _{r \rightarrow \infty}\left(r^{\alpha_{1}} u_{1}, r^{\alpha_{2}} u_{2}\right)=\mathbf{M}^{T}=\left(M_{1}, M_{2}\right) \geq 0 .
$$

Also assume that

$$
\begin{aligned}
& \left(2+\alpha_{1}\right)\left(n-2-\alpha_{1}\right)\left(n-4-2 \alpha_{1}\right) \\
& \quad+\left(2+\alpha_{2}\right)\left(n-2-\alpha_{2}\right)\left(n-4-2 \alpha_{2}\right) \neq 0
\end{aligned}
$$

and

$$
\frac{1}{p+1}+\frac{1}{q+1}>\frac{n-3}{n-1}, \quad \frac{1}{p+1}+\frac{1}{q+1} \neq \frac{n-2}{n} .
$$

Then $\mathbf{u}$ is radially symmetric with respect to some point in $\mathbf{R}^{n}$.

The main ingredients in proving Theorem 1.1 consist of the well-known Alexandrov-Serrin moving plane method (see $[1,8,12,19]$ ) and appropriately strong asymptotics at infinity. When the domain is the entire space, asymptotics play an important role in the procedure; that is, replacing boundary lemmas at infinity and providing a starting point (see for example $[3,5,8,19,21]$ ). We have the following general result.

Theorem 1.2. Let $\mathbf{u}$ be a positive solution of (II). Suppose that the following conditions hold.

$\left(\mathbf{H}_{1}\right)$ There exists $M>0$ such that for $r>M$ and $\left|x_{1}\right|>M$ we have

$$
x_{1} \mathbf{u}_{x_{1}}(x)<0 .
$$

$\left(\mathbf{H}_{2}\right)$ There exist $a_{j}, b_{j}>0$ and $c_{j} \in \mathbf{R}, j=1,2$, such that if $\left\{\delta_{i}\right\} \in \mathbf{R} \rightarrow \delta$ and $\left\{x^{i}\right\} \in \mathbf{R}^{n}$, with $\left|x^{i}\right| \rightarrow \infty, \delta_{i} \neq x_{1}^{i}$, then

$$
\lim _{i \rightarrow \infty} \frac{\left|x^{i}\right|^{a_{j}+2}}{\delta_{i}-x_{1}^{i}}\left(u_{j}\left(x^{i}\right)-u_{j}\left(x^{i^{\delta_{i}}}\right)\right)=2 a_{j} b_{j} \delta-2 c_{j}, \quad j=1,2,
$$

where $x^{i \delta_{i}}$ is the reflection of $x^{i}$ with respect to $x_{i}=\delta_{i}$ with

$$
\gamma_{0} \doteq \frac{c_{1}}{a_{1} b_{1}}=\frac{c_{2}}{a_{2} b_{2}} .
$$

Then $\mathbf{u}$ is symmetric with respect to the hyperplane $x_{1}=\gamma_{0}$.

Remark. Theorem 1.2 holds for more general systems. 
With the aid of Theorem 1.2, one immediately concludes the symmetry by using suitably sharp asymptotics at infinity. Therefore, as in [19], our focus is to establish the following asymptotic expansion.

Theorem 1.3. Let $\mathbf{u}$ be a positive solution of (II). Suppose that the conditions (1.4)-(1.6) hold with $\mathbf{M} \neq 0$. Then we have the following expansion at infinity

$$
u_{j}(x)=r^{-\alpha_{j}}\left(L_{j}+\xi_{j}(r)+\frac{\nu_{j}(r, \theta)}{r}\right), \quad j=1,2,
$$

where $(r, \theta)$ are spherical coordinates. Moreover,

1. For $j=1,2, \xi_{j}(r)=\bar{u}_{j}(r) r^{\alpha_{j}}-L_{j}$, and there exist $k=k(n, p, q)>0$ and $M=M(\mathbf{u}, n, p, q)>0$ such that

$$
\left|\xi_{j}(r)\right| \leq M r^{-k}, \quad\left|\xi_{j}^{\prime}(r)\right| \leq M r^{-k-1}
$$

for $r>0$ large.

2. Let $\tau$ and $\tau_{1}$ be two non-negative integers. Then there exists $M=M\left(\mathbf{u}, \tau, \tau_{1}\right)$ such that

$$
\left|D_{\theta}^{\tau_{1}} D_{r}^{\tau} \nu_{j}\right| \leq M r^{-\tau}, \quad r>1, \quad j=1,2 .
$$

3. Let $\tau$ be a non-negative integer. Then

$$
\lim _{r \rightarrow \infty} \nu_{j}(r, \theta)=k_{j} V(\theta), \quad j=1,2,
$$

uniformly in $C^{\tau}\left(S^{n-1}\right)$, where

$$
\begin{aligned}
& k_{1}=\frac{p L_{2}^{p-1}}{p \alpha_{2}\left(n-2-\alpha_{1}\right)+q \alpha_{1}\left(n-2-\alpha_{2}\right)}>0, \\
& k_{2}=\frac{p \alpha_{2}\left(n-2-\alpha_{1}\right)}{p \alpha_{2}\left(n-2-\alpha_{1}\right)+q \alpha_{1}\left(n-2-\alpha_{2}\right)}>0
\end{aligned}
$$

and

$$
V(\theta)=\frac{x \cdot x_{0}}{r}
$$

for some $x_{0} \in \mathbf{R}^{n}$ fixed.

Such an expansion was obtained in [19] for scalar equations. The key was to study a new equation, via a suitable transform, which has singular coefficients of a critical degree 2. Treating (II) seems somewhat different, namely, difficulties arise partly due to the imbalance between the two exponents $p$ and $q$. For instance, the components $u$ and $v$ of solutions of (II) cannot both have fast decay at infinity everywhere on the critical hyperbola (cf. the fast decay $O\left(r^{2-n}\right)$ when $p=l$ for the Lane-Emden equation). Moreover, the Kelvin transform approach applies only in the square $[0, l] \times[0, l]$ of the $p q$-plane (see $[5])$, rather than in the entire subcritical region below the hyperbola (1.3), as is in the scalar case.

In an 'evolution' setting, Simon [16], [17] studied the question of asymptotics for elliptic systems (see also [2]). It was shown that, with quite general assumptions, bounded solutions must tend to an equilibrium as 'time' $t$ approaches infinity. And moreover, the convergence actually occurs at an exponential 'speed' under further restrictions (see [17] for details). Turning back to system (II), one loses the 'homogeneity'-like structure because of the imbalance between $p$ and $q$, which was used in [17]. Furthermore, due to supercritical growth, there is a structural flaw; 
that is $m<0$ (see [17] for definition), whose opposite plays a crucial role in the derivation of exponential decay in [17]. Therefore a new and different approach is employed. In an effort to extend our results, we shall further develop such asymptotics in a general setting, see a forthcoming paper [22].

It is worth remarking that the comparison results obtained in Section 2 (Lemma 2.1 and Proposition 2.1) seem new and are of independent interest. They can be regarded as generalizations of maximum principles for cooperative systems.

The organization of the paper is as follows. In Section 2, we derive two comparison results (Lemma 2.1 and Proposition 2.1), which are crucial for decay estimates. In Section 3, we obtain better (optimum) bounds for fast decay solutions. Sections 4-6 are devoted to establishing various estimates related to the 'slow decay' solutions $\mathbf{v}$ of (3.8). Indeed, Section 4 contains the derivation of a system of inequalities for the $L^{2}$-average $\mathbf{w}$ of the difference between $\mathbf{v}$ and its $S^{n-1}$-spherical average, while decay estimates are developed for $\mathbf{w}$ and $\overline{\mathbf{v}}$ in Section 5 and for $\mathbf{v}$ itself in Section 6 , respectively. In Section 7 , we establish the crucial asymptotic expansion (Theorem 1.3). Finally, our main theorem (Theorem 1.1) and the general symmetry theorem (Theorem 1.2) will both be proved in Section 8 .

\section{ACKNOWLEDGEMENT}

Part of this paper was written while the author was visiting the University of Perugia, the University of Trieste and Rutgers University. He wishes to thank Professors Brezis, Mitidieri and Pucci for their kind invitations and their institution for the hospitality.

He also wishes to thank the referee for his careful reading of the manuscript and many valuable suggestions, in particular, a question which leads to a much improved Lemma 7.2.

\section{SOME COMPARISON RESULTS}

In this section, we establish two comparison results for a linear two-system of inequalities, which are crucial in developing our asymptotic estimates later on. The proof is based on the classical maximum principle.

Let $\mathbf{A}$ and $\mathbf{B}$ be two $2 \times 2$-matrices, with $\mathbf{A}$ being constant and diagonal, i.e.,

$$
\mathbf{A}=\left(\begin{array}{cc}
a_{1} & 0 \\
0 & a_{2}
\end{array}\right), \quad \mathbf{B}(x)=\left(\begin{array}{cc}
b_{11}(x) & b_{12}(x) \\
b_{21}(x) & b_{22}(x)
\end{array}\right) .
$$

For $\Omega \subset \mathbf{R}^{n}$ open, consider the linear system of inequalities

$$
\Delta \mathbf{u}+\frac{\mathbf{A} \mathbf{u}^{\prime}}{r}+\frac{\mathbf{B}(x) \mathbf{u}}{r^{2}} \geq 0, \quad x \in \Omega,
$$

where $\mathbf{u}^{\prime}$ is the partial derivative of $\mathbf{u}$ with respect to the radius $r$ and

$$
\mathbf{u}^{T}=\left(u_{1}, u_{2}\right) \text {. }
$$

In the sequel throughout the paper, we shall denote by $\mathcal{L}$ the linear operator associated with the system (2.1), namely,

$$
\mathcal{L}(\mathbf{u}) \doteq \Delta \mathbf{u}+\frac{\mathbf{A} \mathbf{u}^{\prime}}{r}+\frac{\mathbf{B}(x) \mathbf{u}}{r^{2}} .
$$

Denote

$$
B_{i j}=\sup _{x \in \Omega} b_{i j}(x), \quad i, j=1,2 .
$$


We shall assume

$$
\max _{i=1,2} B_{i i}<0, \quad b_{i j}(x) \geq 0, \quad x \in \Omega, i \neq j,
$$

and

$$
K_{0}=\operatorname{det}\left(\begin{array}{ll}
B_{11} & B_{12} \\
B_{21} & B_{22}
\end{array}\right)>0
$$

We have the following maximum principle.

Lemma 2.1. Let $\mathbf{u}^{T}(x)=\left(u_{1}(x), u_{2}(x)\right)$ be a solution of (2.1). Suppose $0 \notin \Omega$ and that (2.2) and (2.3) hold and

$$
\left.\mathbf{u}\right|_{x \in \partial \Omega} \leq 0 .
$$

Then

$$
\mathbf{u}(x) \leq 0, \quad x \in \Omega .
$$

Remark. For simplicity, Lemma 2.1 is stated only for (2.1). In fact, the conclusion holds for general $N$-systems. Moreover, a corresponding conclusion holds for general boundary conditions.

Proof. Suppose for contradiction that (2.5) is false, say, $u_{1}\left(x_{0}\right)>0$ for some $x_{0} \in \Omega$. Then $u_{1}$ assumes a positive maximum, say at $x_{0}$. Using $(2.1)_{1}$, one deduces that (noting $\mathbf{A}$ is diagonal)

$$
b_{12}\left(x_{0}\right) u_{2}\left(x_{0}\right) \geq-b_{11}\left(x_{0}\right) u_{1}\left(x_{0}\right) \geq-B_{11} u_{1}\left(x_{0}\right)>0 .
$$

It follows that $u_{2}$ assumes a positive maximum at $x_{1} \in \Omega$. But by $(2.1)_{2}$, one has

$$
b_{21}\left(x_{1}\right) u_{1}\left(x_{1}\right) \geq-b_{22}\left(x_{1}\right) u_{2}\left(x_{1}\right) \geq-B_{22} u_{2}\left(x_{1}\right)>0 .
$$

Therefore, we derive that

$$
B_{12} u_{2}\left(x_{0}\right) \geq-B_{11} u_{1}\left(x_{0}\right), \quad B_{21} u_{1}\left(x_{1}\right) \geq-B_{22} u_{2}\left(x_{1}\right) .
$$

In turn,

$$
B_{12} B_{21} u_{1}\left(x_{1}\right) u_{2}\left(x_{0}\right) \geq B_{11} B_{22} u_{1}\left(x_{0}\right) u_{2}\left(x_{1}\right) .
$$

This is impossible, since

$$
u_{1}\left(x_{0}\right) u_{2}\left(x_{1}\right) \geq u_{1}\left(x_{1}\right) u_{2}\left(x_{0}\right)>0, \quad B_{11} B_{22}>B_{12} B_{21} \geq 0 .
$$

The proof is complete. 
Using Lemma 2.1, we are able to obtain a useful comparison result for such systems.

Proposition 2.1. Let $\mathbf{u}^{T}(x)=\left(u_{1}(x), u_{2}(x)\right)$ be a positive solution of (2.1) on $\Omega=\{r>R\}$ for some $R>0$. Suppose (2.2) and (2.3) hold and

$$
\lim _{r \rightarrow \infty} \mathbf{u}(x)=0 .
$$

Then there exists $k_{0}>0$ such that

$$
\mathbf{u}(x)=O\left(r^{-k}\right), \quad r \rightarrow \infty
$$

for all $k \in\left(0, k_{0}\right)$.

Proof. For $K_{1}, K_{2}>0$ and $k \in \mathbf{R}$, denote

$$
\phi^{T}(x)=\left(\phi_{1}(x), \phi_{2}(x)\right)=\left(K_{1}, K_{2}\right) r^{-k} .
$$

It is by direct calculations

$$
\mathcal{L}(\phi)=\left(\begin{array}{c}
M_{1} \\
M_{2}
\end{array}\right) r^{-k-2}, \quad r>R,
$$

where

$$
\begin{aligned}
M_{1} & =K_{1}\left[k\left(k+2-n-a_{1}\right)+b_{11}(x)\right]+K_{2} b_{12}(x) \\
& \leq K_{1}\left(k^{2}-\left(n-2+a_{1}\right) k+B_{11}\right)+K_{2} B_{12}, \\
M_{2} & =K_{2}\left[k\left(k+2-n+a_{2}\right)+b_{22}(x)\right]+K_{1} b_{21}(x) \\
& \leq K_{2}\left(k^{2}-\left(n-2+a_{2}\right) k+B_{22}\right)+K_{1} B_{21} .
\end{aligned}
$$

Plainly for $c_{1}, c_{2}>0$, by (2.2) and (2.3), the linear system

$$
\left\{\begin{array}{l}
B_{11} K_{1}+B_{12} K_{2}=-c_{1}, \\
B_{21} K_{1}+B_{22} K_{2}=-c_{2}
\end{array}\right.
$$

has a unique positive solution

$$
K_{1}=K_{0}^{-1}\left(B_{12} c_{2}-B_{22} c_{1}\right)>0, \quad K_{2}=K_{0}^{-1}\left(B_{21} c_{2}-B_{11} c_{2}\right)>0 .
$$

It follows from (2.8) that, by making the above choice of $K_{1}$ and $K_{2}$

$$
\begin{aligned}
& M_{1} \leq\left(k^{2}-\left(n-2+a_{1}\right) k\right) K_{1}-c_{1}<0, \\
& M_{2} \leq\left(k^{2}-\left(n-2+a_{2}\right) k\right) K_{2}-c_{2}<0,
\end{aligned}
$$

provided $k \in\left(0, \min \left[k_{1}, k_{2}\right]\right)$, where

$$
\begin{aligned}
& k_{1}=\frac{1}{2}\left(n-2+a_{1}+\sqrt{\left(n-2+a_{1}\right)^{2}+4 c_{1} / K_{1}}\right)>0, \\
& k_{2}=\frac{1}{2}\left(n-2+a_{2}+\sqrt{\left(n-2+a_{2}\right)^{2}+4 c_{2} / K_{2}}\right)>0,
\end{aligned}
$$

respectively, since $K_{1}, K_{2}, c_{1}, c_{2}>0$. It follows, from (2.7), that

$$
\mathcal{L}(\phi)=\left(M_{1}, M_{2}\right)^{T} r^{-k-2}<0, \quad r>R .
$$

Next take $K>0$ large enough so that

and put

$$
\left.(\mathbf{u}-K \phi)\right|_{r=R} \leq 0
$$

$$
\mathbf{Z}^{T}(x)=\left(Z_{1}(x), Z_{2}(x)\right)=\mathbf{u}^{T}-K \phi^{T} .
$$


Therefore, by (2.1) and (2.11), for $r>R$

$$
\mathcal{L}(\mathbf{Z})=\mathcal{L}(\mathbf{u})-K \mathcal{L}(\phi)>0,
$$

and, by (2.6) and the fact $k>0$, clearly

$$
\left.\mathbf{Z}\right|_{r=R} \leq 0, \quad \lim _{r \rightarrow \infty} \mathbf{Z}(x)=0 .
$$

Now Lemma 2.1 implies that $\mathbf{Z} \leq 0$ for $r \geq R$. In particular, our claim follows by taking $k_{0}=\min \left(k_{1}, k_{2}\right)$.

Remark. The choice of $k_{1}$ and $k_{2}$ depends on the choice of $c_{1}$ and $c_{2}$.

\section{Decay estimates: Fast Decay}

In this section, we turn back to study the Lane-Emden system

$$
\Delta \mathbf{u}+\mathbf{f}(\mathbf{u})=0, \quad \mathbf{u}>0, \quad x \in \mathbf{R}^{n},
$$

where

$$
\mathbf{u}=\left(u_{1}, u_{2}\right), \quad \mathbf{f}(\mathbf{u})=\left(u_{2}^{p}, u_{1}^{q}\right), \quad p, q>0 .
$$

Consider solutions of (3.1) satisfying either

$$
u_{1}(x)=o\left(r^{-\alpha_{1}}\right), \quad \text { or } \quad u_{2}(x)=o\left(r^{-\alpha_{2}}\right)
$$

as $r \rightarrow \infty$. We shall refer to such solutions as fast decay solutions. Indeed, one can show that in the case of (3.2), there necessarily holds

$$
u_{1}(x)=O\left(r^{\max (2-n, 2-p(n-2))}\right), \quad u_{2}(x)=O\left(r^{\max (2-n, 2-q(n-2))}\right)
$$

as $r \rightarrow \infty$; see Proposition 3.1. We begin with two elementary lemmas.

Lemma 3.1. Let $\mathbf{u}=\left(u_{1}, u_{2}\right)$ be a positive solution of (3.1). Suppose $p q>1$. Then one has

$$
\bar{u}_{1}(r)=O\left(r^{-\alpha_{1}}\right), \quad \bar{u}_{2}(r)=O\left(r^{-\alpha_{2}}\right)
$$

as $r \rightarrow \infty$, where

$$
\alpha_{1}=\frac{2(p+1)}{p q-1}, \quad \alpha_{2}=\frac{2(q+1)}{p q-1} .
$$

with $\bar{u}_{1}(r)$ and $\bar{u}_{2}(r)$ being the spherical average of $u_{1}$ and $u_{2}$, respectively.

Proof. For the proof, we refer the reader to [14].

Lemma 3.2. Let $\mathbf{u}$ be a positive solution of (3.1). Then one has

$$
\mathbf{u}(x)=\frac{1}{c_{n}} \int \frac{\mathbf{f}(\mathbf{u}(y))}{|x-y|^{n-2}} d y,
$$

where $c_{n}=(n-2) \omega_{n}$ with $\omega_{n}$ the volume of the $(n-1)$-sphere $S^{n-1}$.

Proof. Standard and thus omitted.

Now we are ready to derive asymptotic estimates of positive solutions of (3.1) of fast decay, with the aid of Proposition 2.1. 
Proposition 3.1. Let $\mathbf{u}$ be a positive solution of (3.1). Suppose that (3.2) holds and

$$
p>1, \quad q>1, \quad \max \left(\alpha_{1}, \alpha_{2}\right)<n-2 .
$$

Then

$$
u_{1}(x)=O\left(r^{\max (2-n, 2-p(n-2))}\right), \quad u_{2}(x)=O\left(r^{\max (2-n, 2-q(n-2))}\right)
$$

as $r \rightarrow \infty$.

Remark. When $\max \left(\alpha_{1}, \alpha_{2}\right) \geq n-2$, (3.1) has no non-trivial and non-negative solutions (see $[11,13,14]$ ), while (3.5) guarantees

$$
\min (2-p(n-2), 2-q(n-2))<2-n .
$$

That is, one of the exponents appearing in (3.6) is $2-n$.

Proof. By Lemma 3.2, $(3.2)_{1}$ and $(3.2)_{2}$ are equivalent to each other. That is,

$$
v_{1}(x) \doteq r^{\alpha_{1}} u_{1}(x) \rightarrow 0, \quad v_{2}(x) \doteq r^{\alpha_{2}} u_{2}(x) \rightarrow 0
$$

as $r \rightarrow \infty$. By a direct calculation, $\mathbf{v}=\left(v_{1}, v_{2}\right)$ satisfies

$$
\mathcal{L}(\mathbf{v})=0
$$

where

$$
\mathbf{A}=\left(\begin{array}{cc}
-2 \alpha_{1} & 0 \\
0 & -2 \alpha_{2}
\end{array}\right), \quad \mathbf{B}(x)=\left(\begin{array}{cc}
-\lambda_{1} & v_{2}^{p-1} \\
v_{1}^{q-1} & -\lambda_{2}
\end{array}\right)
$$

Plainly, one has

$$
\begin{gathered}
a_{1}=-2 \alpha_{1}, \quad a_{2}=-2 \alpha_{2}, \\
B_{11}=b_{11}(x)=-\lambda_{1}=-\alpha_{1}\left(n-2-\alpha_{1}\right)<0, \\
B_{22}=b_{22}(x)=-\lambda_{2}=-\alpha_{2}\left(n-2-\alpha_{2}\right)<0,
\end{gathered}
$$

and as $R \rightarrow \infty$,

$$
\begin{aligned}
& 0<B_{12}(R)=\sup _{r>R} b_{12}(x)(R)=\sup _{r>R} v_{2}^{p-1}(x) \rightarrow 0, \\
& 0<B_{21}(R)=\sup _{r>R} b_{21}(x)=\sup _{r>R} v_{1}^{q-1}(x) \rightarrow 0 .
\end{aligned}
$$

Therefore, conditions (2.2), (2.3) and (2.6) hold, and we can apply Proposition 2.1. The choice

$$
c_{1}=-B_{11}, \quad c_{2}=-B_{22}
$$

yields

$K_{1}(R)=K_{0}^{-1}\left(K_{0}+B_{12} \lambda_{2}\right) \rightarrow 1, \quad K_{2}(R)=K_{0}^{-1}\left(K_{0}+B_{21} \lambda_{1}\right) \rightarrow 1, \quad R \rightarrow \infty$.

Using (2.9) and (2.10) for any $\epsilon>0$, there exists $R_{0}>0$ such that for $R>R_{0}$

$$
\begin{aligned}
k_{1} & =\frac{1}{2}\left(n-2+a_{1}+\sqrt{\left(n-2+a_{1}\right)^{2}-4 B_{11} / K_{1}}\right) \\
& =\frac{1}{2}\left(n-2-2 \alpha_{1}+\sqrt{\left(n-2-2 \alpha_{1}\right)^{2}+4 \lambda_{1} / K_{1}}\right) \\
& >\frac{1}{2}\left(n-2-2 \alpha_{1}+\sqrt{\left(n-2-2 \alpha_{1}\right)^{2}+4 \alpha_{1}\left(n-2-\alpha_{1}\right)}\right)-\epsilon \\
& =n-2-\alpha_{1}-\epsilon,
\end{aligned}
$$


and, similarly,

$$
k_{2}=\frac{1}{2}\left(n-2+a_{2}+\sqrt{\left(n-2+a_{2}\right)^{2}-4 B_{22} / K_{2}}\right)>n-2-\alpha_{2}-\epsilon .
$$

It follows that (without loss of generality, assume $p \geq q$ ),

$$
\min \left(k_{1}, k_{2}\right)>n-2-\max \left(\alpha_{1}, \alpha_{2}\right)-\epsilon=n-2-\alpha_{1}-\epsilon>0 .
$$

Therefore, by Proposition 2.1, for any $k \in\left(0, n-2-\alpha_{1}\right)$

$$
v_{1}=O\left(r^{-k}\right), \quad v_{2}=O\left(r^{-k}\right)
$$

since $\epsilon>0$ is arbitrary. In turn,

$$
u_{1}(x)=O\left(r^{-k-\alpha_{1}}\right), \quad u_{2}(x)=O\left(r^{-k-\alpha_{2}}\right) .
$$

A simple calculation yields (recall $p \geq q$ )

$$
p\left(n-2+\alpha_{2}-\alpha_{1}\right)-n=(p-1)\left(n-2-\alpha_{1}\right)>0 .
$$

since $p \alpha_{2}=2+\alpha_{1}$. Using Lemma 3.2, one then deduces that

$$
u_{1}(x)=O\left(r^{2-n}\right), \quad u_{2}(x)=O\left(r^{\max (2-n, 2-q(n-2))}\right),
$$

and the proof is complete.

\section{A System of INEQUALities}

In the next four sections, we shall employ a device introduced in [19] to establish an asymptotic expansion at infinity for solutions $\mathbf{u}$ of (3.1), which is crucial to our moving plane argument.

Put

$$
\mathbf{v}^{T}(x)=\left(v_{1}(x), v_{2}(x)\right)=\left(r^{\alpha_{1}} u_{1}(x), r^{\alpha_{2}} u_{2}(x)\right),
$$

and its spherical average

$$
\overline{\mathbf{v}}^{T}(x)=\left(\bar{v}_{1}(x), \bar{v}_{2}(x)\right)=\left(r^{\alpha_{1}} \bar{u}_{1}(x), r^{\alpha_{2}} \bar{u}_{2}(x)\right) .
$$

Throughout the following four sections, we shall consider solutions satisfying

$$
\lim _{r \rightarrow \infty} \mathbf{v}^{T}(x)=\mathbf{L}^{T}=\left(L_{1}, L_{2}\right)=\left[\left(\lambda_{1} \lambda_{2}^{p}\right)^{1 /(p q-1)},\left(\lambda_{1}^{q} \lambda_{2}\right)^{1 /(p q-1)}\right],
$$

(referred to as slow decay solutions), where, as before,

$$
\alpha_{1}=\frac{2(p+1)}{p q-1}>0, \quad \alpha_{2}=\frac{2(q+1)}{p q-1}>0,
$$

and

$$
\lambda_{1}=\alpha_{1}\left(n-2-\alpha_{1}\right)>0, \quad \lambda_{2}=\alpha_{2}\left(n-2-\alpha_{2}\right)>0 .
$$

Denote

$$
\mathbf{W}^{T}(x)=\left(W_{1}(x), W_{2}(x)\right)=\mathbf{v}^{T}-\overline{\mathbf{v}}^{T}
$$

and

$$
\mathbf{w}^{T}(r)=\left(w_{1}, w_{2}\right)=\left(\left(\int_{S^{n-1}} W_{1}^{2}(r, \theta) d \theta\right)^{1 / 2},\left(\int_{S^{n-1}} W_{2}^{2}(r, \theta) d \theta\right)^{1 / 2}\right),
$$

where $(r, \theta)$ are spherical coordinates.

For any $\delta \in(0,1)$, we first show that $\mathbf{w}(r)$ decays at infinity of rate $\delta$, i.e., $\mathbf{w}(r)=o\left(r^{-\delta}\right)$ as $r \rightarrow \infty$. It follows that $\overline{\mathbf{v}}$ decays at infinity of rate $k$ for some 
$k=k(n, p, q)>0$. Combining above we then show that $\mathbf{W}(x)=O\left(r^{-1}\right)$, and moreover,

$$
r \mathbf{W}^{T}(x) \rightarrow\left(k_{1}, k_{2}\right) V(\theta)
$$

as $r \rightarrow \infty$, where $k_{1}, k_{2}>0$ are given by (1.7) and $V(\theta)$ is a first eigenfunction of $(-\Delta)$ on $S^{n-1}$.

In this section, we derive the following fundamental system of differential inequalities, from which decay estimates of $\mathbf{w}$ can be drawn.

Theorem 4.1. Let $\mathbf{w}$ be given by (4.3). Then for $r>0$ sufficiently large, one has,

$$
\mathcal{L}(\mathbf{w}) \geq 0,
$$

where

$$
\begin{gathered}
\mathbf{A}=\left(\begin{array}{cc}
-2 \alpha_{1} & 0 \\
0 & -2 \alpha_{2}
\end{array}\right), \\
\mathbf{B}(x)=\left(\begin{array}{cc}
-\left(n-1+\lambda_{1}\right) & p L_{2}^{p-1}+2 F(r) \\
q L_{1}^{q-1}+2 G(r) & -\left(n-1+\lambda_{2}\right)
\end{array}\right)
\end{gathered}
$$

with

$$
F(r)=\max _{|x|=r}\left|p v_{2}^{p-1}(x)-p L_{2}^{p-1}\right| \rightarrow 0, \quad G(r)=\max _{|x|=r}\left|q v_{1}^{q-1}(x)-q L_{1}^{q-1}\right| \rightarrow 0
$$

as $r \rightarrow \infty$.

Proof. It amounts to showing $(4.4)_{1}$, with $(4.4)_{2}$ being essentially the same. Take the spherical average of (3.8) and then the difference between the result and (3.8)

$$
\Delta \mathbf{W}+\frac{\mathbf{A W}^{\prime}}{r}+\frac{\mathbf{B}(x) \mathbf{v}-\overline{\mathbf{B}(x) \mathbf{v}}}{r^{2}}=0,
$$

where $\mathbf{A}$ and $\mathbf{B}$ are given by (3.9), namely,

$$
\mathbf{A}=\left(\begin{array}{cc}
-2 \alpha_{1} & 0 \\
0 & -2 \alpha_{2}
\end{array}\right), \quad \mathbf{B}(x)=\left(\begin{array}{cc}
-\lambda_{1} & v_{2}^{p-1} \\
v_{1}^{q-1} & -\lambda_{2}
\end{array}\right) .
$$

Thus $W_{1}$ satisfies the equation

$$
\Delta W_{1}-\frac{2 \alpha_{1} W_{1}^{\prime}}{r}-\frac{\lambda_{1} W_{1}}{r^{2}}+\frac{v_{2}^{p}-\overline{v_{2}^{p}}}{r^{2}}=0 .
$$

Using spherical coordinates $(r, \theta)$, we multiply by $W_{1}$ and integrate over $S^{n-1}$ to get

$$
\begin{aligned}
\int_{S^{n-1}} W_{1}^{\prime \prime} W_{1} & +\frac{n-1-2 \alpha_{1}}{r} \int_{S^{n-1}} W_{1}^{\prime} W_{1}-\frac{\lambda_{1}}{r^{2}} \int_{S^{n-1}} W_{1}^{2} \\
& +\frac{1}{r^{2}} \int_{S^{n-1}} W_{1} \Delta_{\theta} W_{1}+\frac{1}{r^{2}} \int_{S^{n-1}}\left[v_{2}^{p}-\overline{v_{2}^{p}}\right] W_{1}=0 .
\end{aligned}
$$

We shall estimate (4.6) term by term. Clearly one has

$$
\int_{S^{n-1}} W_{1}^{\prime} W_{1}=w_{1} w_{1}^{\prime}, \quad w_{1}^{\prime 2} \leq \int_{S^{n-1}} W_{1}^{\prime 2} .
$$

Therefore,

$$
\int_{S^{n-1}} W_{1}^{\prime \prime} W_{1}+\int_{S^{n-1}} W_{1}^{\prime 2}=w_{1} w_{1}^{\prime \prime}+w_{1}^{\prime 2} \leq w_{1} w_{1}^{\prime \prime}+\int_{S^{n-1}} W_{1}^{\prime 2},
$$


and in turn

$$
\int_{S^{n-1}} W_{1}^{\prime \prime} W_{1} \leq w_{1} w_{1}^{\prime \prime}
$$

Using the Wirtinger inequality, integration by parts gives

$$
\int_{S^{n-1}} W_{1} \Delta_{\theta} W_{1}=-\int_{S^{n-1}}\left|\nabla_{\theta} W_{1}\right|^{2} \leq-(n-1) \int_{S^{n-1}} W_{1}^{2}=-(n-1) w_{1}^{2}
$$

since $\bar{W}_{1}=0$. To estimate the last term in (4.6), we have

$$
\begin{aligned}
\int_{S^{n-1}} & {\left[v_{2}^{p}-\overline{v_{2}^{p}}\right] W_{1} } \\
= & \int_{S^{n-1}}\left[v_{2}^{p}-p L_{2}^{p-1} v_{2}-\left(\overline{v_{2}^{p}}-p L_{2}^{p-1} \bar{v}_{2}\right)+p L_{2}^{p-1} W_{2}\right] W_{1} \\
= & \int_{S^{n-1}}\left[f\left(v_{2}\right)-\overline{f\left(v_{2}\right)}\right] W_{1}+p L_{2}^{p-1} \int_{S^{n-1}} W_{1} W_{2},
\end{aligned}
$$

where

$$
f(t)=t^{p}-p L_{2}^{p-1} t .
$$

By the mean value theorem, there exists a $\xi=\xi(x)$ between $v_{2}(x)$ and $\bar{v}_{2}(r)$ such that

$$
f\left(v_{2}\right)-f\left(\bar{v}_{2}\right)=f^{\prime}(\xi)\left(v_{2}-\bar{v}_{2}\right)=f^{\prime}(\xi) W_{2}
$$

Note that

$$
\lim _{r \rightarrow \infty} f^{\prime}(\xi)=\lim _{r \rightarrow \infty} p\left(\xi^{p-1}-L_{2}^{p-1}\right)=0
$$

by assumption (4.1), and

$$
\overline{f\left(v_{2}\right)}-f\left(\bar{v}_{2}\right)=\frac{1}{\omega_{n-1}} \int_{S^{n-1}}\left(f\left(v_{2}\right)-f\left(\bar{v}_{2}\right)\right)=\frac{-1}{\omega_{n-1}} \int_{S^{n-1}} f^{\prime}(\xi) W_{2} .
$$

It follows that, with the aid of the Schwartz inequality,

$$
\begin{aligned}
& \left|\int_{S^{n-1}}\left[f\left(v_{2}\right)-\overline{f\left(v_{2}\right)}\right] W_{1}\right| \\
& \quad=\mid \int_{S^{n-1}}\left[\left(f\left(v_{2}\right)-f\left(\bar{v}_{2}\right)\right)-\left(\overline{f\left(v_{2}\right)}-f\left(\bar{v}_{2}\right)\right] W_{1} \mid\right. \\
& \quad \leq \int_{S^{n-1}}\left|f\left(v_{2}\right)-f\left(\bar{v}_{2}\right)\right| \cdot\left|W_{1}\right|+\int_{S^{n-1}} \mid\left(\overline{f\left(v_{2}\right)}-f\left(\bar{v}_{2}\right)|\cdot| W_{1} \mid\right. \\
& \quad \leq F(r) \int_{S^{n-1}}\left|W_{1} W_{2}\right|+\frac{F(r)}{\omega_{n}} \int_{S^{n-1}}\left|W_{1}\right| \cdot \int_{S^{n-1}}\left|W_{2}\right| \\
& \quad \leq 2 F(r) w_{1} w_{2},
\end{aligned}
$$

where

$$
F(r)=\max _{|x|=r}\left|f^{\prime}(\xi)\right| \rightarrow 0, \quad \text { as } r \rightarrow \infty .
$$

Combining (4.10) and (4.11), we obtain

$$
\left|\int_{S^{n-1}}\left[v_{2}^{p}-\overline{v_{2}^{p}}\right] W_{1}\right| \leq\left(p L_{2}^{p-1}+2 F(r)\right) w_{1} w_{2}
$$


Substituting (4.7)-(4.9) and (4.12) into (4.6), we obtain

$$
w_{1} w_{1}^{\prime \prime}+\frac{n-1-2 \alpha_{1}}{r} w_{1} w_{1}^{\prime}-\frac{n-1+\lambda_{1}}{r} w_{1}^{2}+\frac{p L_{2}^{p-1}+2 F(r)}{r} w_{1} w_{2} \geq 0 .
$$

Dividing both sides by $w_{1}$, one gets $(4.4)_{1}\left((4.4)_{2}\right.$ similarly $)$ and the proof is complete.

\section{Decay estimates: Slow decay-Radial}

In this section, we shall develop decay estimates for $\mathbf{w}(r)$ and $\overline{\mathbf{v}}(r)$ at infinity. The following fundamental hypothesis

$$
\frac{1}{p+1}+\frac{1}{q+1}>\frac{n-3}{n-1}
$$

comes right into play.

Theorem 5.1. Let $\mathbf{w}$ be given by (4.3), thus satisfying (4.4). Suppose that $(p, q)$ is subcritical with respect to the dimension $n-1$, i.e., (5.1) holds. Then for all $k \in(0,1)$

$$
\mathbf{w}(r)=O\left(r^{-k}\right)
$$

as $r \rightarrow \infty$.

Proof. By Theorem 4.1, w satisfies (4.4) with A and B given by (4.5); namely,

$$
\begin{gathered}
a_{1}=-2 \alpha_{1}, \quad a_{2}=-2 \alpha_{2}, \\
B_{11}=-\left(n-1+\lambda_{1}\right)<0, \quad B_{22}=-\left(n-1+\lambda_{2}\right)<0,
\end{gathered}
$$

and

$$
B_{12}=p L_{2}^{p-1}+2 \sup _{r>R} F(r) \rightarrow p L_{2}^{p-1}, \quad B_{21}=q L_{1}^{q-1}+2 \sup _{r>R} G(r) \rightarrow q L_{1}^{q-1}
$$

as $R \rightarrow \infty$. Clearly there exists $R_{0}>0$ such that for $R>R_{0}$

$$
\begin{aligned}
K_{0}(R)=\operatorname{det} \mathbf{B} & =B_{11} B_{22}-B_{12} B_{21} \\
& =\left(n-1+\lambda_{1}\right)\left(n-1+\lambda_{2}\right)-p q \lambda_{1} \lambda_{2}+o(1)>0,
\end{aligned}
$$

provided that

$$
\left(n-1+\lambda_{1}\right)\left(n-1+\lambda_{2}\right)-p q \lambda_{1} \lambda_{2}>0,
$$

which is equivalent to (5.1) by a simple calculation. Hence the conditions (2.2) and (2.3) are satisfied (clearly (2.6) ia satisfied by (4.1)), and Proposition 2.1 is applicable. Therefore, for all $k \in\left(0, \min \left[k_{1}, k_{2}\right]\right)$, where $k_{1}>0$ and $k_{2}>0$ are given by (2.9) and (2.10), respectively (e.g., taking $c_{1}=-B_{11}$ and $c_{2}=-B_{22}$ ), there holds,

$$
\mathbf{w}(r)=O\left(r^{-k}\right), \quad r \rightarrow \infty .
$$

Denote

$$
k_{0}=\sup \{k \in(0,1) \mid(5.2) \text { holds }\} .
$$

We claim $k_{0}=1$.

Suppose for contradiction that $k_{0}<1$. For $\sigma \in(0,1)$, put

$$
\hat{\mathbf{w}}(r)=r^{\sigma} \mathbf{w}(r) .
$$


One readily verifies that $\hat{\mathbf{w}}(r)$ satisfies the system (4.4), while $\mathbf{A}$ and $\mathbf{B}$ have the entries

$$
\begin{gathered}
a_{1}=-2 \sigma-2 \alpha_{1}, \quad a_{2}=-2 \sigma-2 \alpha_{2}, \\
B_{11}=-\left(n-1+\lambda_{1}\right)-\sigma\left(n-2-\sigma-2 \alpha_{1}\right)<0,
\end{gathered}
$$

and

$$
B_{22}=-\left(n-1+\lambda_{2}\right)-\sigma\left(n-2-\sigma-2 \alpha_{2}\right)<0,
$$

(with $B_{12}$ and $B_{21}$ unchanged), and moreover,

$$
\begin{aligned}
D(\sigma) & =\left[\left(n-1+\lambda_{1}\right)+\sigma\left(n-2-\sigma-2 \alpha_{1}\right)\right]\left[\left(n-1+\lambda_{2}\right)+\sigma\left(n-2-\sigma-2 \alpha_{2}\right)\right] \\
& >p q \lambda_{1} \lambda_{2},
\end{aligned}
$$

if (5.1) holds. Therefore

$$
K_{0}=\operatorname{det} \mathbf{B}=D(\sigma)-p q \lambda_{1} \lambda_{2}+o(1)>0
$$

for $\sigma \in(0,1)$. In particular, for $\sigma \in\left(0, k_{0}\right)$, we may apply the previous argument to $\hat{\mathbf{w}}(r)$ to obtain

$$
\hat{\mathbf{w}}(r)=O\left(r^{-k}\right), \quad r \rightarrow \infty
$$

for all $k \in\left(0, \min \left[k_{1}, k_{2}\right]\right)$. Clearly $k_{1}>0$ and $k_{2}>0$ can be chosen depending only on $k_{0}<1$ (i.e., independent of $\sigma$ ) for all $\sigma \in\left(0, k_{0}\right)$. It follows that

$$
\hat{\mathbf{w}}(r)=O\left(r^{-k_{0}-\epsilon}\right), \quad r \rightarrow \infty,
$$

for some $\epsilon>0$, a contradiction and the proof is complete.

Abusing notation, denote

$$
\mathbf{v}^{T}=\mathbf{v}^{T}-\left(L_{1}, L_{2}\right) .
$$

Using the decay estimate of $\mathbf{w}$ obtained in the last section, we see that $\overline{\mathbf{v}}$ satisfies an 'almost' linear system.

Lemma 5.1. The spherical average $\overline{\mathbf{v}}$ satisfies the following nonhomogeneous system

$$
\overline{\mathbf{v}}^{\prime \prime}+\mathbf{A} \overline{\mathbf{v}}^{\prime}+\mathbf{B} \overline{\mathbf{v}}=O\left(|\overline{\mathbf{v}}|^{2}\right)+h(t)
$$

where $t=\ln r$,

$$
\mathbf{A}=\left(\begin{array}{cc}
n-2-2 \alpha_{1} & 0 \\
0 & n-2-2 \alpha_{2}
\end{array}\right), \quad \mathbf{B}=\left(\begin{array}{cc}
-\lambda_{1} & p L_{2}^{p-1} \\
q L_{1}^{q-1} & -\lambda_{2}
\end{array}\right),
$$

and for all $k \in(0,1)$

$$
\begin{aligned}
|h(t)| \leq & \left|\left(\bar{v}_{2}+L_{2}\right)^{p}-\overline{\left(v_{2}+L_{2}\right)^{p}}\right| \\
& +\left|\left(\bar{v}_{1}+L_{1}\right)^{q}-\overline{\left(v_{1}+L_{1}\right)^{q}}\right|=O(|\mathbf{w}|)=O\left(e^{-k t}\right)
\end{aligned}
$$

as $t \rightarrow \infty$.

Proof. It is by direct calculations, with the aid of Theorem 5.1.

Using Lemma 5.1, we immediately derive the asymptotic behavior of $\overline{\mathbf{v}}$ at infinity. 
Theorem 5.2. Suppose $\alpha_{1}+\alpha_{2} \neq n-2$. Then there exists $k=k(n, p, q)>0$ such that

$$
\overline{\mathbf{v}}=O\left(r^{-k}\right)
$$

as $r \rightarrow \infty$.

Remark. The condition $\alpha_{1}+\alpha_{2} \neq n-2$ is equivalent to

$$
\frac{1}{p+1}+\frac{1}{q+1} \neq \frac{n-2}{n} \text {. }
$$

Proof. We first convert the system (5.4) into a first order system by introducing

$$
\mathbf{x}^{T}=\left(x_{1}, x_{2}, x_{3}, x_{4}\right), \quad x_{1}=\bar{v}_{1}, \quad x_{2}=\bar{v}_{1}^{\prime}, \quad x_{3}=\bar{v}_{2}, \quad x_{4}=\bar{v}_{2}^{\prime} .
$$

Then $\mathbf{x}$ satisfies the system

$$
\mathbf{x}^{\prime}=\mathbf{A} \mathbf{x}+O\left(|\mathbf{x}|^{2}\right)+h(t),
$$

where $h(t)$ satisfies $(5.5)$ and

$$
\mathbf{A}=\left(\begin{array}{cccc}
0 & 1 & 0 & 0 \\
\lambda_{1} & -\left(n-2-2 \alpha_{1}\right) & -p L_{2}^{p-1} & 0 \\
0 & 0 & 0 & 1 \\
-q L_{1}^{q-1} & 0 & \lambda_{2} & -\left(n-2-2 \alpha_{2}\right)
\end{array}\right)
$$

It is easy to check that the characteristic polynomial of $\mathbf{A}$ is given by

$$
\begin{aligned}
c(m)= & m^{4}+2 D m^{3}+\left[\left(n-2-2 \alpha_{1}\right)\left(n-2-2 \alpha_{2}\right)-\lambda_{1}-\lambda_{2}\right] m^{2} \\
& -\left[\lambda_{1}\left(n-2-2 \alpha_{2}\right)+\lambda_{2}\left(n-2-2 \alpha_{1}\right)\right] m-(p q-1) \lambda_{1} \lambda_{2} \\
= & m^{4}+2 D m^{3}+\left(D^{2}-\left(\alpha_{1}+\alpha_{2}\right) D-\alpha_{1}^{2}-\alpha_{2}^{2}\right) m^{2} \\
& -\left[\lambda_{1}+\lambda_{2}+\left(\alpha_{1}-\alpha_{2}\right)^{2}\right] D m-(p q-1) \lambda_{1} \lambda_{2} .
\end{aligned}
$$

where

$$
D=D\left(\alpha_{1}, \alpha_{2}\right)=n-2-\alpha_{1}-\alpha_{2} .
$$

Thus one readily sees that all roots of $c(m)$ have nonzero real part provided that

$$
D=n-2-\alpha_{1}-\alpha_{2} \neq 0, \quad \text { i.e., } \quad n-2 \neq \alpha_{1}+\alpha_{2} .
$$

Therefore, without loss of generality (by changing coordinates), we assume

$$
\mathbf{A}=\left(\begin{array}{cc}
\mathbf{A}_{1} & 0 \\
0 & \mathbf{A}_{2}
\end{array}\right)
$$

where there exists $\sigma>0$ such that

$$
e^{\mathbf{A}_{1} t} \leq e^{-\sigma t}, \quad t \geq 0 ; \quad e^{\mathbf{A}_{2} t} \leq e^{\sigma t}, \quad t \leq 0 .
$$

Using the variation of parameters, we solve (5.7) for $\mathbf{x}$ for $t>t_{0}$

$$
\begin{aligned}
\mathbf{x}(t)= & e^{\mathbf{A}_{1}\left(t-t_{0}\right)} \mathbf{x}\left(t_{0}\right)+\int_{t_{0}}^{t} e^{\mathbf{A}_{1}(t-s)}\left[h_{1}(s)+O\left(|\mathbf{x}|^{2}\right)\right] d s \\
& -\int_{t}^{\infty} e^{\mathbf{A}_{2}(t-s)}\left[h_{2}(s)+O\left(|\mathbf{x}|^{2}\right)\right] d s,
\end{aligned}
$$


which is justified since $|\mathbf{x}| \rightarrow 0$ as $t \rightarrow \infty$. It follows that

$$
\begin{aligned}
|\mathbf{x}(t)| \leq & \left|e^{\mathbf{A}_{1}\left(t-t_{0}\right)} \mathbf{x}\left(t_{0}\right)\right|+\int_{t_{0}}^{t}\left|e^{\mathbf{A}_{1}(t-s)}\left[h_{1}(s)+O\left(|\mathbf{x}|^{2}\right)\right]\right| d s \\
& +\int_{t}^{\infty}\left|e^{\mathbf{A}_{1}(t-s)}\left[h_{2}(s)+O\left(|\mathbf{x}|^{2}\right)\right]\right| d s \\
\leq & c e^{-\sigma t}+\int_{t_{0}}^{t} e^{-\sigma(t-s)}\left[h_{1}(s)+O\left(|\mathbf{x}|^{2}\right)\right] d s \\
& +\int_{t}^{\infty} e^{\sigma(t-s)}\left[h_{2}(s)+O\left(|\mathbf{x}|^{2}\right)\right] d s \\
\leq & c\left(e^{-\sigma t}+e^{-k t}\right)+\int_{t_{0}}^{t} e^{-\sigma(t-s)}\left[O\left(|\mathbf{x}|^{2}\right)\right] d s+\int_{t}^{\infty} e^{\sigma(t-s)}\left[O\left(|\mathbf{x}|^{2}\right)\right] d s
\end{aligned}
$$

For $\epsilon>0$, there exists $t_{0}>0$ such that

$$
|\mathbf{x}(t)|<\epsilon, \quad t \geq t_{0}
$$

It follows that

$$
e^{\sigma t}|\mathbf{x}(t)| \leq c\left(1+e^{(\sigma-k) t}\right)+\epsilon \int_{t_{0}}^{t} e^{\sigma s}|\mathbf{x}| d s+2 \sigma \epsilon \int_{t}^{\infty} e^{\sigma(2 t-s)}|\mathbf{x}| d s .
$$

Put

$$
x(t)=\int_{t_{0}}^{t} e^{\sigma s}|\mathbf{x}(s)| d s \geq 0, \quad y(t)=\int_{t}^{\infty} e^{2 \sigma t-\sigma s}|\mathbf{x}(s)| d s \geq 0,
$$

and

$$
z(t)=x(t)+y(t)
$$

Plainly,

$$
z^{\prime}(t)=x^{\prime}(t)+y^{\prime}(t)=e^{\sigma t}|\mathbf{x}(t)|-e^{\sigma t}|\mathbf{x}(t)|+2 \sigma \int_{t}^{\infty} e^{2 \sigma t-\sigma s}|\mathbf{x}(s)| d s=2 \sigma y(t) .
$$

It follows that from (5.8),

$$
x^{\prime}(t) \leq c\left(1+e^{(\sigma-k) t}\right)+\epsilon x(t)+2 \sigma \epsilon y(t),
$$

and in turn,

$$
x^{\prime}(t)-2 \epsilon x(t)-4 \sigma \epsilon y(t) \leq c\left(1+e^{(\sigma-k) t}\right)-\epsilon x(t)-2 \sigma \epsilon y(t)
$$

or rather,

$$
x^{\prime}(t)-2 \epsilon x(t)-2 \epsilon z^{\prime}(t) \leq c\left(1+e^{(\sigma-k) t}\right)-\epsilon x(t)-2 \sigma \epsilon y(t) .
$$

Add $4 \epsilon^{2} z(t)$ to both sides to get

$$
\begin{aligned}
x^{\prime}(t) & -2 \epsilon x(t)-2 \epsilon z^{\prime}(t)+4 \epsilon^{2} z(t) \\
& \leq c\left(1+e^{(\sigma-k) t}\right)-\epsilon x(t)-2 \sigma \epsilon y(t)+4 \epsilon^{2} z(t) \\
& =c\left(1+e^{(\sigma-k) t}\right)-\epsilon x(t)-2 \sigma \epsilon y(t)+4 \epsilon^{2} x(t)+4 \epsilon^{2} y(t) \\
& \leq c\left(1+e^{(\sigma-k) t}\right),
\end{aligned}
$$

provided that $\epsilon$ is chosen sufficiently small. Rewrite the left-hand side to get

$$
\left(e^{-2 \epsilon t}[x(t)-2 \epsilon z(t)]\right)^{\prime} \leq c\left(e^{-2 \epsilon t}+e^{(\sigma-2 \epsilon-k) t}\right) .
$$


Integrate from $t_{0}$ to $t>t_{0}$ to get

$$
e^{-2 \epsilon t}(x(t)-2 \epsilon z(t)) \leq c\left(1+e^{(\sigma-2 \epsilon-k) t}\right)+e^{-2 \epsilon t_{0}}\left(x\left(t_{0}\right)-2 \epsilon z\left(t_{0}\right)\right) .
$$

But

$$
e^{-2 \epsilon t_{0}}\left(x\left(t_{0}\right)-2 \epsilon z\left(t_{0}\right)\right)=-2 \epsilon e^{-2 \epsilon t_{0}} y\left(t_{0}\right)<0,
$$

since $x\left(t_{0}\right)=0$. Finally, we arrive at

$$
x(t) \leq c\left(e^{2 \epsilon t}+e^{(\sigma-k) t}\right)+2 \epsilon z(t) .
$$

Putting (5.9) into (5.8) yields

$$
e^{-\sigma t}|\mathbf{x}(t)| \leq c \epsilon\left(e^{2(\epsilon-\sigma) t}+e^{-(\sigma+k) t}\right)+2(\sigma+\epsilon) \epsilon \int_{t}^{\infty} e^{-\sigma s}|\mathbf{x}(s)| d s .
$$

Now the standard Gronwall inequality immediately implies (for $\epsilon$ sufficiently small)

$$
e^{-\sigma t}|\mathbf{x}(t)| \leq c\left(e^{2(\epsilon-\sigma) t}+e^{-(\sigma+k) t}\right) .
$$

In particular,

$$
|\mathbf{x}(t)| \leq c\left(e^{(2 \epsilon-\sigma) t}+e^{-k t}\right) .
$$

It is clear that (5.6) is equivalent to (5.10) after changing variables back and the proof is complete.

\section{Decay estimates: Slow Decay-Non-Radial}

Utilizing the decay estimates for both $\overline{\mathbf{v}}$ and $\mathbf{w}$ obtained in last section, we are able to apply a local maximum principle to obtain the desired decay estimates for $\mathbf{v}$ and $\mathbf{W}$ as well as similar estimates for their derivatives.

We first have the following local maximum principle for elliptic systems.

Lemma 6.1. Let $\Omega \subset \mathbf{R}^{n}$ be an open domain. Suppose that

$$
\mathbf{u}(x)=\left(u^{1}(x), u^{2}(x), \cdots, u^{N}(x)\right) \in\left(W^{2, n}(\Omega)\right)^{N}
$$

satisfies the system

$$
a_{i j}^{l}(x) u_{i j}^{l}+b_{i}^{l}(x) u_{i}^{l}+c_{i}^{l}(x) u^{i}=h^{l}(x), \quad x \in \Omega, \quad l=1,2, \cdots, N,
$$

where $\mathbf{h} \in\left(L^{n}(\Omega)\right)^{N}$. Assume that there exist three positive numbers $k, k_{1}$ and $k_{2}$ such that

$$
k|\xi|^{2} \leq a_{i j}^{l}(x) \xi_{i} \xi_{j} \leq k_{1}|\xi|^{2}, \quad \xi \in \mathbf{R}^{n}, \quad l=1,2, \cdots, N
$$

and

$$
\left|b^{l}\right| \leq k^{2} k_{2}, \quad\left|c^{l}\right| \leq k k_{2}, \quad l=1,2, \cdots, N
$$

where

$$
\left|b^{l}\right|=\max _{x \in \Omega}\left(\sum_{i=1}^{n}\left(b_{i}^{l}(x)\right)^{2}\right), \quad\left|c^{l}\right|=\max _{x \in \Omega}\left(\sum_{i=1}^{n}\left|c_{i}^{l}(x)\right|\right) .
$$

Then for any ball $B=B_{2 R}(y) \subset \Omega$ and $q>0$, we have

$$
\sup _{x \in B_{R}(y)}|\mathbf{u}(x)| \leq C\left\{\left(\frac{1}{|B|} \int_{B}|\mathbf{u}|^{q}\right)^{1 / q}+\frac{R}{k}\|\mathbf{h}\|_{L^{n}(B)}\right\},
$$

where $C=C\left(n, q, k_{1} / k, k_{2} R^{2}\right)$. 
Lemma 6.1 is proved for the scalar case in [9, Theorem 9.20, p. 244] and the proof involves the so-called 'upper contact set'. Since only 'mixed terms' appearing in (6.1) are of zero order, the proof remains virtually applicable (de-couple) with little modification and we thus omit it.

Theorem 6.1. Let $\tau>0$ be an integer, $\mathbf{v}(x)$ and $\mathbf{W}(x)$ given by (5.3) and (4.2), respectively. Then there exists a constant $M=M(\mathbf{v}, n, p, q, \tau)>0$ such that for some $k=k(n, p, q)>0$

$$
\max _{|x|=r}\left|D^{\tau} \mathbf{v}(x)\right| \leq M r^{-k-\tau}
$$

and for all $k \in(0,1)$

$$
\max _{|x|=r}\left|D^{\tau} \mathbf{W}(x)\right| \leq M r^{-k-\tau} .
$$

Proof. Clearly, by Theorems 5.1 and $5.2, \mathbf{v}(x)$ and $\mathbf{W}(x)$ satisfy the systems

$$
\Delta \mathbf{v}+\frac{\mathbf{A v}^{\prime}}{r}+\frac{\mathbf{B}(x) \mathbf{v}}{r^{2}}=0
$$

and

$$
\Delta \mathbf{W}+\frac{\mathbf{A W}^{\prime}}{r}+\frac{\mathbf{B}_{1}(x) \mathbf{W}}{r^{2}}=O\left(r^{-k-3}\right)
$$

respectively, where $k=k(n, p, q)>0$,

$$
\begin{gathered}
\mathbf{A}=\left(\begin{array}{cc}
-2 \alpha_{1} & 0 \\
0 & -2 \alpha_{2}
\end{array}\right), \\
\mathbf{B}(x)=\left(\begin{array}{c}
-\lambda_{1} \\
v_{1}^{-1}\left[\left(v_{1}+L_{1}\right)^{q}-L_{1}^{q}\right]
\end{array} \quad \begin{array}{c}
v_{2}^{-1}\left[\left(v_{2}+L_{2}\right)^{p}-L_{2}^{p}\right] \\
-\lambda_{2}
\end{array}\right) \in L^{\infty}
\end{gathered}
$$

and

$$
\mathbf{B}_{1}(x)=\left(\begin{array}{cc}
-\lambda_{1} & W_{2}^{-1}\left[\left(v_{2}+L_{2}\right)^{p}-\left(\bar{v}_{2}+L_{2}\right)^{p}\right] \\
W_{1}^{-1}\left[\left(v_{1}+L_{1}\right)^{q}-\left(\bar{v}_{1}+L_{1}\right)^{q}\right] & -\lambda_{2}
\end{array}\right) \in L^{\infty}
$$

In particular, Lemma 6.1 applies. Similarly, as in [19], it suffices to deduce that for $x_{0}$ and $\tau \geq 0$, there exists $M>0$ such that

$$
\int_{B_{r_{0} / 2}}\left|D^{\tau} \mathbf{v}(x)\right|^{2} \leq M r_{0}^{n-2 k-2 \tau}
$$

for some $k=k(n, p, q)>0$, and

$$
\int_{B_{r_{0} / 2}}\left|D^{\tau} \mathbf{W}(x)\right|^{2} \leq M r_{0}^{n-2 k-2 \tau}
$$

for all $k \in(0,1)$, where $r_{0}=\left|x_{0}\right| / 2>0$ and $B=B_{r_{0}}\left(x_{0}\right)$. But, for example, say $\tau=0$, one has

$$
\begin{aligned}
\int_{B_{r_{0} / 2}}|\mathbf{v}(x)|^{2} & \leq 2 \int_{B_{r_{0} / 2}}\left(|\mathbf{W}|^{2}+|\overline{\mathbf{v}}|^{2}\right) \leq \int_{B_{3 r_{0} / 2}(0) \backslash B_{r_{0} / 2}(0)}\left(|\mathbf{W}|^{2}+|\overline{\mathbf{v}}|^{2}\right) \\
& \leq c r_{0}^{n} \max _{r \geq r_{0} / 2}\left(|\mathbf{w}|^{2}+|\overline{\mathbf{v}}|^{2}\right)=c r_{0}^{n} \max _{r \geq r_{0} / 2}|\overline{\mathbf{v}}|^{2}=O\left(r_{0}^{n-2 k}\right)
\end{aligned}
$$

and

$$
\int_{B_{r_{0} / 2}}|\mathbf{W}|^{2} \leq \int_{B_{3 r_{0} / 2}(0) \backslash B_{r_{0} / 2}(0)}|\mathbf{W}|^{2} \leq c r_{0}^{n} \max _{r \geq r_{0} / 2}|\mathbf{w}|^{2}=O\left(r_{0}^{n-2 k}\right) .
$$


The estimates (6.4) can actually be refined further. In fact, we have the following result.

Theorem 6.2. Let $\tau \geq 0$ be an integer. Then there exists a constant $M=$ $M(\mathbf{v}, \tau)>0$ (independent of $r$ ) such that

$$
\max _{|x|=r}\left|D^{\tau} \mathbf{W}(x)\right| \leq M r^{-1-\tau} .
$$

The proof of Theorem 6.2 is essentially the same as that of Theorem 6.1, which is a consequence of the local maximum principle and the following local $L^{2}$-estimate for $\mathbf{W}$ near infinity.

Lemma 6.2. There exists a constant $M=M(\mathbf{v})>0$ such that

$$
\int_{B}\left|D^{\tau} \mathbf{W}\right|^{2} \leq M r_{0}^{n-2-2 \tau},
$$

where $B=B_{r_{0} / 2}\left(x_{0}\right)$ and $r_{0}=\left|x_{0}\right|>0$.

Proof. It suffices to prove (6.6) for $\tau=0$. By the definitions of $\mathbf{W}$ and $\mathbf{w}$, we need to show

$$
\mathbf{w}(r)=O\left(r^{-1}\right), \quad r \rightarrow \infty .
$$

From (4.4), by Theorems 5.1 and 5.2, we have

$$
\mathcal{L}(\mathbf{w}) \geq O\left(r^{-3-\delta}\right), \quad r>0
$$

for some $\delta \in(0,1)$, where

$$
\mathbf{A}=\left(\begin{array}{cc}
-2 \alpha_{1} & 0 \\
0 & -2 \alpha_{2}
\end{array}\right), \quad \mathbf{B}=\left(\begin{array}{cc}
-\left(n-1+\lambda_{1}\right) & p L_{2}^{p-1} \\
q L_{1}^{q-1} & -\left(n-1+\lambda_{2}\right)
\end{array}\right) .
$$

For $K_{1}, K_{2}, k \in \mathbf{R}$, put

$$
\phi_{k}^{T}(r)=\left(K_{1}, K_{2}\right) r^{-k}
$$

It is by direct calculations that

$$
\mathcal{L}\left(\phi_{k}\right)=B(k)\left(K_{1}, K_{2}\right)^{T} r^{-2-k},
$$

where

$$
B(k)=\left(\begin{array}{cc}
-\left(n-1+\lambda_{1}\right)-k\left(n-2-k-2 \alpha_{1}\right) & p L_{2}^{p-1} \\
q L_{1}^{q-1} & -\left(n-1+\lambda_{2}\right)-k\left(n-2-k-2 \alpha_{2}\right)
\end{array}\right) .
$$

Plainly there exists a $k_{0} \in[1+\delta / 3,1+2 \delta / 3]$ such that

$$
\operatorname{det}\left(B\left(k_{0}\right)\right) \neq 0 \text {. }
$$

Therefore, the system

$$
B\left(k_{0}\right)\left(\begin{array}{l}
K_{1} \\
K_{2}
\end{array}\right)=\left(\begin{array}{l}
-1 \\
-1
\end{array}\right)
$$

has a unique nontrivial solution $\mathbf{K}_{\mathbf{0}}{ }^{T}=\left(K_{1}^{0}, K_{2}^{0}\right)$. In turn

$$
\mathcal{L}\left(\phi_{k_{0}}\right)=\left(\begin{array}{c}
-1 \\
-1
\end{array}\right) r^{-2-k_{0}}, \quad \phi_{k_{0}}=\mathbf{K}_{\mathbf{0}} r^{-k_{0}} .
$$

Note that

$$
b_{11}=-p \alpha_{2}\left(n-2-\alpha_{1}\right)<0, \quad b_{12}=p L_{2}^{p-1}>0
$$


and

$$
\operatorname{det}(B(1))=p \alpha_{2}\left(n-2-\alpha_{1}\right) \cdot q \alpha_{1}\left(n-2-\alpha_{2}\right)-p L_{2}^{p-1} \cdot q L_{1}^{q-1}=0 .
$$

It follows that there exist $K_{1}^{1}>0$ and $K_{2}^{1}>0$ such that

$$
\mathcal{L}\left(\phi_{1}\right)=\mathbf{0}, \quad \phi_{1}=\left(\begin{array}{c}
K_{1}^{1} \\
K_{2}^{1}
\end{array}\right) r^{-1} .
$$

Fix $r_{0} \gg 1$, and take $K>0$ large enough so that

$$
\left.\left(K \phi_{1}+\phi_{k_{0}}\right)\right|_{r=r_{0}}>0 .
$$

For $K_{0}>0$, denote

$$
\Phi(r)=\mathbf{w}(r)-K_{0}\left(K \phi_{1}+\phi_{k_{0}}\right) .
$$

Choose $K_{0}$ so large that both

$$
\left.\Phi(r)\right|_{r=r_{0}} \leq 0
$$

and

$$
\begin{aligned}
\mathcal{L}(\Phi) & =\mathcal{L}(\mathbf{w})-K_{0} K \mathcal{L}\left(\phi_{1}\right)-K_{0} \mathcal{L}\left(\phi_{k_{0}}\right) \\
& =\mathcal{L}(\mathbf{w})+K_{0}\left(\begin{array}{c}
1 \\
1
\end{array}\right) r^{-2-k_{0}} \geq O\left(r^{-3-\delta}\right)+K_{0}\left(\begin{array}{c}
1 \\
1
\end{array}\right) r^{-3-2 \delta / 3}>0 .
\end{aligned}
$$

Thus by Lemma 2.1, we have

$$
\Phi(r) \leq 0, \quad \text { for } r \geq r_{0},
$$

since

$$
\lim _{r \rightarrow \infty} \Phi(r)=0
$$

The proof is complete.

\section{An ASYMPTOTIC EXPANSION}

In this section, we are going to complete the last step to obtain the desired expansion. Introduce the function

$$
\tilde{\mathbf{W}}(r, \theta)=r \mathbf{W}(r, \theta),
$$

where $\mathbf{W}$ is given in (4.2). We shall show that $\tilde{\mathbf{W}}(r, \theta)$ approaches $\mathbf{k} V(\theta)$ as $r \rightarrow$ $\infty$, where $\mathbf{k}$ is given by (1.5) and $V(\theta)$ is a first eigenfunction of $-\Delta_{\theta}$ on $S^{n-1}$. Consequently, we obtain an expansion of $\mathbf{v}$ in terms of $\overline{\mathbf{v}}$ with the 'good' remainder $\tilde{\mathbf{W}}$.

The proof is divided into several technical lemmas and is similar to that of [19]. Therefore, we shall omit most of the proofs.

Lemma 7.1. Let $\tilde{\mathbf{W}}$ be given by (7.1). Then for any non-negative integers $\tau$ and $\tau_{1}$, there exists a constant $M=M\left(\tau, \tau_{1}\right)>0$ such that

$$
\left|D_{\theta}^{\tau_{1}} D_{r}^{\tau} \tilde{\mathbf{W}}\right| \leq M r^{-\tau}, \quad r \geq M
$$

Moreover, $\tilde{\mathbf{W}}$ satisfies the system

$$
\mathcal{L}(\tilde{\mathbf{W}})=O\left(r^{-2-k}\right), \quad r>0
$$

for some $k \in(0,1)$, where

$$
\mathbf{A}=\left(\begin{array}{cc}
-2-2 \alpha_{1} & 0 \\
0 & -2-2 \alpha_{2}
\end{array}\right)
$$


and

$$
\mathbf{B}=\left(\begin{array}{cc}
-\left(\lambda_{1}+n-3-2 \alpha_{1}\right) & p L_{2}^{p-1} \\
q L_{1}^{q-1} & -\left(\lambda_{2}+n-3-2 \alpha_{2}\right)
\end{array}\right) .
$$

Proof. This lemma is a direct consequence of Theorems 6.1 and 6.2.

The following lemma follows from Lemma 7.1.

Lemma 7.2. There holds

$$
r \int_{S^{n-1}}\left|\tilde{\mathbf{W}}^{\prime}\right|^{2} \in L^{1}(1, \infty), \quad r^{3} \int_{S^{n-1}}\left|\tilde{\mathbf{W}}^{\prime \prime}\right|^{2} \in L^{1}(1, \infty) .
$$

Proof. The idea is similar to that for single equations (see [19]), with a more complicated proof. We shall only prove the first inequality. Multiply $(7.3)_{1}$ by $r \tilde{W}_{1}$, $(7.3)_{2}$ by $r \tilde{W}_{2}$ and integrate over $S^{n-1}$ and from $r$ to $R>r$ to obtain

$$
\begin{aligned}
\int_{r}^{R} & \int_{S^{n-1}}\left\{\left[\left|\nabla_{\theta} \tilde{W}_{1}\right|^{2}-b_{11} \tilde{W}_{1}^{2}-b_{12} \tilde{W}_{1} \tilde{W}_{2}\right] / s+s \tilde{W}^{\prime}{ }_{1}^{2}\right\} \\
& =\left.\int_{S^{n-1}}\left[s \tilde{W}_{1} \tilde{W}_{1}^{\prime}+\frac{n-2+a_{1}}{2} \tilde{W}_{1}^{2}\right]\right|_{r} ^{R}+\int_{r}^{R} O\left(s^{-1-k}\right) \int_{S^{n-1}} \tilde{W}_{1}
\end{aligned}
$$

and

$$
\begin{aligned}
\int_{r}^{R} \int_{S^{n-1}}\left\{\left[\left|\nabla_{\theta} \tilde{W}_{2}\right|^{2}-b_{22} \tilde{W}_{2}^{2}-b_{21} \tilde{W}_{1} \tilde{W}_{2}\right] / s+s \tilde{W}_{2}^{\prime 2}\right\} \\
=\left.\int_{S^{n-1}}\left[s \tilde{W}_{2} \tilde{W}_{2}^{\prime}+\frac{n-2+a_{2}}{2} \tilde{W}_{2}^{2}\right]\right|_{r} ^{R}+\int_{r}^{R} O\left(s^{-1-k}\right) \int_{S^{n-1}} \tilde{W}_{2} .
\end{aligned}
$$

Multiply the first equality by $b_{21}$ and the second by $b_{12}$ and add them to

$$
\begin{aligned}
\int_{r}^{R} & \int_{S^{n-1}}\left[s \sum_{i=1}^{2} c \tilde{W}_{i}^{\prime}{ }_{i}^{2}+s^{-1} H(s)\right]=\left.\int_{S^{n-1}} \sum_{i=1}^{2} c s \tilde{W}_{i} \tilde{W}_{i}^{\prime}\right|_{r} ^{R} \\
& +\left.\int_{S^{n-1}}\left[\frac{b_{21}\left(n-2+a_{1}\right)}{2} \tilde{W}_{1}^{2}+\frac{b_{12}\left(n-2+a_{2}\right)}{2} \tilde{W}_{2}^{2}\right]\right|_{r} ^{R}+O\left(r^{-k}\right),
\end{aligned}
$$

where $c=c\left(a_{i}, b_{i j}\right)>0$ (may vary from one to another) and

$$
\begin{array}{r}
H(s)=\int_{S^{n-1}}\left[b_{21}\left(\left|\nabla_{\theta} \tilde{W}_{1}\right|^{2}-(n-1) \tilde{W}_{1}^{2}\right)+b_{12}\left(\left|\nabla_{\theta} \tilde{W}_{2}\right|^{2}-(n-1) \tilde{W}_{2}^{2}\right)\right. \\
\left.+b_{21}\left(n-1-b_{11}\right) \tilde{W}_{1}^{2}+b_{12}\left(n-1-b_{22}\right) \tilde{W}_{2}^{2}-2 b_{12} b_{21} \tilde{W}_{1} \tilde{W}_{2}\right],
\end{array}
$$

Clearly all the terms on the right-hand side of (7.4) are bounded for all $R>r$ by Lemma 7.1 .

Obviously,

$$
b_{12}>0, b_{21}>0, n-1-b_{11}>0, n-1-b_{22}>0 .
$$

It follows that

$$
\int_{S^{n-1}}\left[b_{21}\left(\left|\nabla_{\theta} \tilde{W}_{1}\right|^{2}-(n-1) \tilde{W}_{1}^{2}\right)+b_{12}\left(\left|\nabla_{\theta} \tilde{W}_{2}\right|^{2}-(n-1) \tilde{W}_{2}^{2}\right)\right] \geq 0
$$

by Wirtinger's inequality, since

$$
\tilde{W}_{1}=\bar{W}_{2}=0
$$

Moreover,

$$
b_{21}\left(n-1-b_{11}\right) \cdot b_{12}\left(n-1-b_{22}\right)-\left(b_{12} b_{21}\right)^{2}=0 .
$$


In turn

$$
b_{21}\left(n-1-b_{11}\right) \tilde{W}_{1}^{2}+b_{12}\left(n-1-b_{22}\right) \tilde{W}_{2}^{2}-2 b_{12} b_{21} \tilde{W}_{1} \tilde{W}_{2} \geq 0 .
$$

Therefore,

$$
H(r) \geq 0 \quad \text { for } r>0 .
$$

It follows that from (7.4)

$$
r \int_{S^{n-1}}\left|\tilde{\mathbf{W}}^{\prime}\right|^{2}, r^{-1} H(r) \in L^{1}(1, \infty) .
$$

The proof is complete.

Using Lemma 7.2, one has the following two limits.

Lemma 7.3. Let $\tau$ be a non-negative integer. Then we have

$$
\lim _{r \rightarrow \infty} r \tilde{\mathbf{W}}^{\prime}(r, \theta)=0, \quad \lim _{r \rightarrow \infty} r^{2} \tilde{\mathbf{W}}^{\prime \prime}(r, \theta)=0
$$

in $C^{\tau}\left(S^{n-1}\right)$ uniformly.

After these technical preparations, we are able to prove the main result of this section.

Theorem 7.1. Let $\tilde{\mathbf{W}}$ be a solution of (7.3). Suppose

$$
\begin{aligned}
& \left(2+\alpha_{1}\right)\left(n-2-\alpha_{1}\right)\left(n-4-2 \alpha_{1}\right) \\
& \quad+\left(2+\alpha_{2}\right)\left(n-2-\alpha_{2}\right)\left(n-4-2 \alpha_{2}\right) \neq 0 .
\end{aligned}
$$

Then there holds

$$
\lim _{r \rightarrow \infty} \tilde{\mathbf{W}}(r, \theta)=\left(\begin{array}{c}
k_{1} \\
k_{2}
\end{array}\right) V(\theta),
$$

where $k_{1}$ and $k_{2}$ are given by (1.7), and $V$ is a first eigenfunction of $-\Delta$ on $S^{n-1}$ (with eigenvalue $n-1$ ), i.e.,

$$
\Delta_{\theta} V+(n-1) V=0, \quad \bar{V}=0 .
$$

Proof. First, fix a sequence $\left\{r_{j}\right\} \rightarrow \infty$. Using Lemmas 7.1 and 7.3, we have

$$
\lim _{j \rightarrow \infty} \tilde{W}_{i}\left(r_{j}, \theta\right)=V_{i}(\theta), \quad i=1,2,
$$

in $C^{\tau}\left(S^{n-1}\right)$ for any fixed $\tau \geq 0$ (subject to a subsequence, with $V_{1}$ and $V_{2}$ possibly depending on the sequence) where

$$
\begin{aligned}
& \Delta_{\theta} V_{1}(\theta)+b_{11} V_{1}(\theta)+b_{12} V_{2}(\theta)=0, \\
& \Delta_{\theta} V_{2}(\theta)+b_{21} V_{1}(\theta)+b_{22} V_{2}(\theta)=0 .
\end{aligned}
$$

We shall show that $V_{1}$ and $V_{2}$ are independent of the choice of the sequence. Following the same idea of proving Theorem 7.1 in [19], we put (abusing notation)

$$
\tilde{\mathbf{W}}^{T}(r, \theta)=\tilde{\mathbf{W}}^{T}(r, \theta)-\left(V_{1}, V_{2}\right),
$$

and we want to show that

$$
\lim _{r \rightarrow \infty} \tilde{\mathbf{W}}(r, \theta)=0
$$


Multiply $(7.3)_{1}$ by $r^{2} \tilde{W}_{1}^{\prime}$ and $(7.3)_{2}$ by $r^{2} \tilde{W}_{2}^{\prime}$, and integrate over $S^{n-1}$ and from $r$ to $R>r$ to obtain

$$
\begin{aligned}
\int_{S^{n-1}} & \left.\left(\left|\nabla_{\theta} \tilde{W}_{1}\right|^{2}-b_{11} \tilde{W}_{1}^{2}\right)\right|_{r} ^{R}-2 b_{12} \int_{r}^{R} \int_{S^{n-1}} \tilde{W}_{2} \tilde{W}_{1}^{\prime} \\
= & \left.\left(s^{2} \int_{S^{n-1}} \tilde{W}_{1}^{\prime}{ }_{1}^{2}\right)\right|_{r} ^{R}+\int_{r}^{R} \int_{S^{n-1}}\left[2\left(n-2+a_{1}\right) s \tilde{W}_{1}^{\prime 2}+O\left(s^{-k}\right) \tilde{W}_{1}^{\prime}\right]
\end{aligned}
$$

and

$$
\begin{aligned}
\int_{S^{n-1}} & \left.\left(\left|\nabla_{\theta} \tilde{W}_{2}\right|^{2}-b_{22} \tilde{W}_{2}^{2}\right)\right|_{r} ^{R}-2 b_{21} \int_{r}^{R} \int_{S^{n-1}} \tilde{W}_{1} \tilde{W}_{2}^{\prime} \\
= & \left.\left(s^{2} \int_{S^{n-1}} \tilde{W}_{2}^{\prime}\right)\right|_{r} ^{R}+\int_{r}^{R} \int_{S^{n-1}}\left[2\left(n-2+a_{2}\right) s \tilde{W}_{2}^{\prime}+O\left(s^{-k}\right) \tilde{W}_{2}^{\prime}\right],
\end{aligned}
$$

where $a_{i}$ and $b_{i j}$ are entries of $\mathbf{A}$ and $\mathbf{B}$, respectively. Multiplying the first equality by $b_{21}$ and the second by $b_{12}$ and adding them together yields

$$
\begin{aligned}
\left.H(s)\right|_{r} ^{R}= & \left.b_{21}\left(s^{2} \int_{S^{n-1}} \tilde{W}_{1}^{\prime 2}\right)\right|_{r} ^{R}+b_{21} \int_{r}^{R} \int_{S^{n-1}}\left[2\left(n-2+a_{1}\right) s \tilde{W}_{1}^{\prime}+O\left(s^{-k}\right) \tilde{W}_{1}^{\prime}\right] \\
& +\left.b_{12}\left(s^{2} \int_{S^{n-1}} \tilde{W}_{2}^{\prime}\right)\right|_{r} ^{R}+b_{12} \int_{r}^{R} \int_{S^{n-1}}\left[2\left(n-2+a_{2}\right) s \tilde{W}_{2}^{\prime 2}+O\left(s^{-k}\right) \tilde{W}_{2}^{\prime}\right],
\end{aligned}
$$

where $H(r)$ is given in (7.5). With the aid of Lemma 7.3 and (7.9), let $R \rightarrow \infty$ along the sequence $\left\{r_{j}\right\}$ to obtain

$$
\begin{aligned}
H(r)= & b_{21} r^{2} \int_{S^{n-1}} \tilde{W}_{1}^{2}-b_{21} \int_{r}^{\infty} \int_{S^{n-1}}\left[\left(n-2+a_{1}\right) s \tilde{W}_{1}^{\prime 2}+O\left(s^{-k}\right) \tilde{W}_{1}^{\prime}\right] \\
& +b_{12} r^{2} \int_{S^{n-1}} \tilde{W}_{2}^{\prime 2}-b_{12} \int_{r}^{\infty} \int_{S^{n-1}}\left[2\left(n-2+a_{2}\right) s \tilde{W}_{2}^{\prime}{ }_{2}^{2}+O\left(s^{-k}\right) \tilde{W}_{2}^{\prime}\right] .
\end{aligned}
$$

Therefore, by Lemmas 7.2 and 7.3 ,

$$
H(r)=o(1), \quad \text { as } r \rightarrow \infty .
$$

It follows that,

$$
\begin{aligned}
0 & \leq \int_{S^{n-1}}\left[b_{21}\left(n-1-b_{11}\right) \tilde{W}_{1}^{2}+b_{12}\left(n-1-b_{22}\right) \tilde{W}_{2}^{2}-2 b_{12} b_{21} \tilde{W}_{1} \tilde{W}_{2}\right] \\
& \leq H(r)=o(1)
\end{aligned}
$$

as $r \rightarrow \infty$. Therefore, by (7.6)

$$
\int_{S^{n-1}}\left[b_{21}\left(n-1-b_{11}\right) \tilde{W}_{1}^{2}-b_{12}\left(n-1-b_{22}\right) \tilde{W}_{2}^{2}\right]=o(1) .
$$

On the other hand, letting $R \rightarrow \infty$ along the sequence $\left\{r_{j}\right\}$ in (7.4) yields

$$
\begin{aligned}
\int_{S^{n-1}} & {\left[\frac{b_{21}\left(n-2+a_{1}\right)}{2} \tilde{W}_{1}^{2}+\frac{b_{12}\left(n-2+a_{2}\right)}{2} \tilde{W}_{2}^{2}\right](r) } \\
= & r \int_{S^{n-1}} \sum_{i=1}^{2} c \tilde{W}_{i} \tilde{W}_{i}^{\prime}(r)-\int_{r}^{\infty} s \int_{S^{n-1}} \sum_{i=1}^{2} c \tilde{W}_{i}{ }_{i}^{2} \\
& -\int_{r}^{\infty} s^{-1} H+O\left(r^{-k}\right)=o(1)
\end{aligned}
$$

as $r \rightarrow \infty$. 
Combining (7.11) and (7.12), one concludes the proof of (7.10) since by (7.7)

$$
\begin{array}{r}
b_{21}\left(n-1-b_{11}\right) b_{12}\left(n-2+a_{2}\right)+b_{12}\left(n-1-b_{22}\right) b_{21}\left(n-2+a_{1}\right) \\
=b_{12} b_{21}\left[\left(2+\alpha_{1}\right)\left(n-2-\alpha_{1}\right)\left(n-4-2 \alpha_{1}\right)\right. \\
\left.+\left(2+\alpha_{2}\right)\left(n-2-\alpha_{2}\right)\left(n-4-2 \alpha_{2}\right)\right] \neq 0 .
\end{array}
$$

Now put

$$
\hat{W}(r, \theta)=\frac{b_{21}}{n-1-b_{11}} \tilde{W}_{1}+\tilde{W}_{2} .
$$

By (7.3), $\hat{W}(r, \theta)$ satisfies the equation

$$
\Delta_{\theta} \hat{W}+(n-1) \hat{W}=r h_{1}\left(\tilde{\mathbf{W}}^{\prime}\right)+r^{2} h_{2}\left(\tilde{\mathbf{W}}^{\prime \prime}\right)+O\left(r^{-2-k}\right),
$$

where $h_{1}$ and $h_{2}$ are smooth functions of their arguments and satisfy

$$
h_{i}(\mathbf{Z})=O(|\mathbf{Z}|), \quad i=1,2,
$$

as $|\mathbf{Z}| \rightarrow 0$. Similarly, as in proving (7.10), letting $r \rightarrow \infty$ in (7.13), one has

$$
\lim _{r \rightarrow \infty} \hat{W}(r, \theta)=V(\theta)
$$

uniformly in $C^{\tau}$ for some $V(\theta)$ satisfying (7.8). Clearly

$$
\frac{b_{21}}{n-1-b_{11}} V_{1}+V_{2}=V \text {. }
$$

In other words,

$$
\Delta_{\theta} V_{1}(\theta)+b_{11} V_{1}(\theta)+b_{12}\left(V(\theta)-\frac{b_{21}}{n-1-b_{11}} V_{1}\right)=0 .
$$

Obviously,

$$
b_{11}-\frac{b_{12} b_{21}}{n-1-b_{11}}<n-1 .
$$

Thus the equation (7.14) has a unique solution

$$
V_{1}(\theta)=\frac{b_{12} V(\theta)}{2 n-2-b_{11}-b_{22}} .
$$

Consequently,

$$
V_{2}(\theta)=V(\theta)-\frac{b_{21} V_{1}(\theta)}{n-1-b_{11}}=\frac{\left(n-1-b_{11}\right) V(\theta)}{2 n-2-b_{11}-b_{22}} .
$$

Now one readily verifies that $k_{1}$ and $k_{2}$ satisfy (1.7) and the proof is complete.

We are able now to establish the desired asymptotic expansion at infinity for slow decay solutions of (I), which enables us to proceed with the moving plane procedure. The following explicit formula of the first eigenfunctions of $-\Delta$ on $S^{n-1}$ also plays a role.

Lemma 7.4 (First eigenfunctions of $-\Delta$ on $S^{n-1}$, [4]). Let $V(\theta)$ be a solution of the eigenvalue problem

$$
\Delta_{\theta} V+(n-1) V=0, \quad \bar{V}=\frac{1}{\omega_{n}} \int_{S^{n-1}} V(\theta) d \theta=0 .
$$


Then necessarily $V$ has the form

$$
V=\frac{x}{r} \cdot x_{0}
$$

for some $x_{0} \in \mathbf{R}^{n}$ fixed, where $x \in \mathbf{R}^{n}$.

Simply collecting the results obtained in Sections $4-7$, one immediately proves the asymptotic expansion formula, Theorem 1.3.

\section{THE SYMMETRY}

In this section, we apply the well-known Alexandrov-Serrin moving plane method (see $[1,8,12,19])$ to establish the desired radial symmetry for solutions of (I) with an algebraic decay at infinity. Specifically, consider the system

$$
\Delta \mathbf{u}+\mathbf{f}(\mathbf{u})=0, \quad \mathbf{u}>0, \quad x \in \mathbf{R}^{n},
$$

where

$$
\mathbf{u}^{T}=\left(u_{1}, u_{2}\right), \quad \mathbf{f}^{T}(\mathbf{u})=\left(u_{2}^{p}, u_{1}^{q}\right), \quad p, q>1 .
$$

Assume

$$
\lim _{r \rightarrow \infty} \mathbf{v}^{T}(x)=\lim _{r \rightarrow \infty}\left(r^{\alpha_{1}} u_{1}, r^{\alpha_{2}} u_{2}\right)=\mathbf{M}^{T}=\left(M_{1}, M_{2}\right) \geq 0 .
$$

We shall show that every solution of (8.1) satisfying (8.2) is radially symmetric provided that

$$
\begin{aligned}
& \left(2+\alpha_{1}\right)\left(n-2-\alpha_{1}\right)\left(n-4-2 \alpha_{1}\right) \\
& \quad+\left(2+\alpha_{2}\right)\left(n-2-\alpha_{2}\right)\left(n-4-2 \alpha_{2}\right) \neq 0
\end{aligned}
$$

and

$$
\frac{1}{p+1}+\frac{1}{q+1}>\frac{n-3}{n-1}, \quad \frac{1}{p+1}+\frac{1}{q+1} \neq \frac{n-2}{n} .
$$

For $\gamma \in \mathbf{R}$, let $\Sigma_{\gamma}$ be the hyperplane

$$
\Sigma_{\gamma}=\left\{x=\left(x_{1}, \cdots, x_{n}\right) \in \mathbf{R}^{n}: x_{1}=\gamma\right\} .
$$

For $x \in \mathbf{R}^{n}$, denote $x^{\gamma}$ the reflection point of $x$ about $\Sigma_{\gamma}$; that is,

$$
x^{\gamma}=\left(2 \gamma-x_{1}, x_{2}, \cdots, x_{n}\right) .
$$

As mentioned in the introduction, the asymptotics play an important role in showing symmetry. Indeed, appropriately sharp asymptotics at infinity guarantees the desired symmetry via moving planes; see Theorem 1.2. In order to prove the theorem, we need the following special form of maximum principles, whose proof is standard, exactly the same as that for scalar cases.

Lemma 8.1. Let $\gamma \in \mathbf{R}$ and $\mathbf{u}$ be a positive solution of (8.1). Suppose that

$$
\mathbf{u}(x) \geq \mathbf{u}\left(x^{\gamma}\right), \quad \mathbf{u}(x) \not \equiv u\left(x^{\gamma}\right), \quad \text { if } x_{1}<\gamma .
$$

Then

$$
\mathbf{u}(x)>\mathbf{u}\left(x^{\gamma}\right), \quad \text { if } x_{1}<\gamma
$$

and

$$
\frac{\partial \mathbf{u}(x)}{\partial x_{1}}<0, \quad \text { on } x_{1}=\gamma,
$$

where $x^{\gamma}$ is the reflection point of $x$ with respect to $\Sigma_{\gamma}$. 
Proof of Theorem 1.2. The proof is similar to that for scalar equations (cf. [19, Sec. 10]). We first claim that there exists $\gamma^{\prime}>0$ such that

$$
\mathbf{u}(x)>\mathbf{u}\left(x^{\gamma}\right), \quad \text { if } x_{1}<\gamma \text { and } \gamma \geq \gamma^{\prime} .
$$

Suppose for contradiction that (8.5) is not true. Then there exist two sequences $\left\{\gamma^{i}\right\} \rightarrow \infty$ and $\left\{x^{i}\right\}$ with $x_{1}^{i}<\gamma^{i}$ such that

$$
\mathbf{u}\left(x^{i}\right) \ngtr \mathbf{u}\left(y^{i}\right), \quad y^{i}=x^{i^{\gamma^{i}}}, \quad i=1,2, \cdots,
$$

say (without loss of generality),

$$
u_{1}\left(x^{i}\right) \leq u_{1}\left(y^{i}\right), \quad i=1,2, \cdots .
$$

Obviously $\left|y^{i}\right| \rightarrow \infty$, so $u_{1}\left(y^{i}\right) \rightarrow 0$. In turn $\left|x^{i}\right| \rightarrow \infty$. By $\left(\mathbf{H}_{1}\right)$, we must have

$$
x_{1}^{i} \leq M, \quad \text { for } i \text { large. }
$$

It follows that for any $\gamma_{1}>\max \left(\gamma_{0}, M\right)$

$$
u_{1}\left(x^{i}\right) \leq u_{1}\left(y^{i}\right) \leq u_{1}\left(x^{i^{\gamma_{1}}}\right), \quad \text { for } i \text { large, }
$$

where $\gamma_{0}$ is in Theorem 1.2. On the other hand, by $\left(\mathbf{H}_{2}\right)$, one has

$$
0 \geq \frac{\left|x^{i}\right|^{\alpha_{j}+2}}{\gamma_{1}-x_{1}^{i}}\left[u_{1}\left(x^{i}\right)-u_{1}\left(x^{i^{\gamma_{1}}}\right)\right] \rightarrow 2 a_{1} b_{1} \gamma_{1}-2 c_{1}>0 .
$$

This is a contradiction and (8.5) follows.

Now let $\Gamma$ be a subset of $\mathbf{R}$ defined by

$$
\Gamma=\left\{\gamma \in\left(\gamma_{0}, \infty\right):(8.5) \text { holds }\right\} .
$$

We shall prove that

$$
\Gamma=\left(\gamma_{0}, \infty\right) .
$$

We first claim that $\Gamma$ is open. Otherwise, for some $\gamma \in \Gamma$, there exist two sequences $\left\{\gamma^{i}\right\} \rightarrow \gamma$ and $\left\{x^{i}\right\}$ with $x_{1}^{i} \neq \gamma^{i}$ such that (8.6) holds. Obviously there is a subsequence of $\left\{x^{i}\right\}$ tending to either infinity or $z \in \mathbf{R}^{n}$ as $i \rightarrow \infty$. If the first case occurs, we simply use $\left(\mathbf{H}_{2}\right)$ to derive a contradiction as before, since $\gamma>\gamma_{0}$. If the second case happens, we infer, from the definition of $\gamma$, that

$$
z_{1}=\gamma .
$$

It follows that

$$
\mathbf{u}(x)>\mathbf{u}\left(x^{\gamma}\right), \quad \text { if } x_{1}<\gamma,
$$

and (say, using (8.7) option),

$$
\frac{\partial u_{1}}{\partial x_{1}}(z) \geq 0 .
$$

This simply cannot happen by Lemma 8.1 , that is, $\Gamma$ is open.

Put

$$
\widetilde{\gamma}=\inf \left\{\gamma \in\left(\gamma_{0}, \infty\right):(\gamma, \infty) \subset \Gamma\right\} .
$$

Clearly $\widetilde{\gamma}<\infty$ is finite. We want to show that

$$
\widetilde{\gamma}=\gamma_{0} \text {. }
$$

Suppose for contradiction this is not true, i.e., $\widetilde{\gamma}>\gamma_{0}$. By continuity, one has

$$
\mathbf{u}(x) \geq \mathbf{u}\left(x^{\widetilde{\gamma}}\right) \quad \text { for } x_{1}<\widetilde{\gamma} .
$$


Thanks to Lemma 8.1, one sees that either

$$
\mathbf{u}(x) \equiv \mathbf{u}\left(x^{\widetilde{\gamma}}\right) \quad \text { for } x_{1}<\widetilde{\gamma}
$$

or

$$
\mathbf{u}\left(x^{i}\right)>\mathbf{u}\left(x^{\widetilde{\gamma}}\right) \quad \text { for } x_{1}<\widetilde{\gamma}, \quad \text { i.e., } \quad \widetilde{\gamma} \in \Gamma \text {. }
$$

The latter cannot occur because $(\widetilde{\gamma}, \infty)$ is maximal and $\Gamma$ is open. The former cannot occur either because it contradicts $\left(\mathbf{H}_{2}\right)$ since $\widetilde{\gamma}>\gamma_{0}$. Thus $\widetilde{\gamma}=\gamma_{0}$ and (8.8) is proved.

By continuity again, we have

$$
\mathbf{u}(x) \geq \mathbf{u}\left(x^{\gamma_{0}}\right) \quad \text { for } x_{1}<\gamma_{0} .
$$

Reversing the $x_{1}$-axis (i.e., $\left.\left(x_{1}, x^{\prime}\right) \rightarrow\left(-x_{1}, x^{\prime}\right)\right)$, we conclude that

$$
\mathbf{u}(x) \geq \mathbf{u}\left(x^{-\gamma_{0}}\right) \quad \text { for } x_{1}>\gamma_{0} .
$$

That is, $u$ is symmetric about the hyperplane $x_{1}=\gamma_{0}$ and the proof is complete.

We now can prove our symmetry theorem.

Theorem 8.1. Let $\mathbf{u}$ be a positive solution of (8.1). Suppose that the conditions (8.2)-(8.4) hold. Then $\mathbf{u}$ is necessarily radial symmetric with respect to some point in $\mathbf{R}^{n}$.

Proof. Apparently, by $(8.3)_{3}$, we have either

or

$$
\frac{1}{p+1}+\frac{1}{q+1}>\frac{n-2}{n}
$$

$$
\frac{n-2}{n}>\frac{1}{p+1}+\frac{1}{q+1}>\frac{n-3}{n-1} .
$$

We divide the proof into three cases.

(i) First, consider

$$
\frac{1}{p+1}+\frac{1}{q+1}>\frac{n-2}{n} .
$$

Then $\mathbf{u} \equiv 0$ by a result of $[13$, see Theorem 1.1] and there is nothing left to prove.

(ii) Next, assume either $M_{1}=0$ or $M_{2}=0$, and

$$
\frac{n-2}{n}>\frac{1}{p+1}+\frac{1}{q+1}>\frac{n-3}{n-1} .
$$

By Proposition 3.1, we have

$$
u_{1}(x)=O\left(r^{\max (2-n, 2-p(n-2))}\right), \quad u_{2}(x)=O\left(r^{\max (2-n, 2-q(n-2))}\right) .
$$

Without loss of generality, assume $p \geq q$, whence

$$
u_{1}(x)=O\left(r^{2-n}\right), \quad u_{2}(x)=O\left(r^{\max (2-n, 2-q(n-2))}\right) .
$$

In particular, the arguments of [13, Theorem 1.1] carry over with little modification since one can easily check that

$$
(q+1)(2-n)<-n
$$


and

$$
(p+1) \max (2-n, 2-q(n-2))<-n
$$

since (8.9) holds. Therefore, $\mathbf{u} \equiv 0$ and we are done.

(iii) Finally, consider the case $M_{1}, M_{2}>0$ and (8.9) holds. We shall apply the moving plane method, with the aid of Theorem 1.2. In order to apply Theorem 1.2, we need to derive the asymptotics $\left(\mathbf{H}_{1}\right)$ and $\left(\mathbf{H}_{2}\right)$. Using the expansion formula Theorem 1.3, we have the following result.

Lemma 8.2. Let $\mathbf{u}$ be a positive solution of (I). Suppose that conditions of Theorem 8.1 hold with $M_{1}, M_{2}>0$. Then

(i) there holds the limit

$$
\lim _{r \rightarrow \infty} \mathbf{v}^{T}(x)=\lim _{r \rightarrow \infty}\left(r^{\alpha_{1}} u_{1}(x), r^{\alpha_{2}} u_{2}(x)\right)=\left(L_{1}, L_{2}\right) .
$$

(ii) there exists $M>0$ such that for $r,\left|x_{1}\right|>M$

$$
x_{1} \mathbf{u}_{x_{1}}(x)<0 .
$$

(iii) if $l_{i} \in \mathbf{R} \rightarrow l$ and $\left\{x^{i}\right\}$ is a sequence of points going to infinity, with $x_{1}^{i} \neq l_{i}$, then

$$
\lim _{i \rightarrow \infty} \frac{\left|x^{i}\right|^{\alpha_{j}+2}}{l_{i}-x_{1}^{i}}\left(u_{j}\left(x^{i}\right)-u_{j}\left(x^{i^{l_{i}}}\right)\right)=2 \alpha_{j} L_{j} l-2 k_{j}\left(x_{0}\right)_{1}, \quad j=1,2,
$$

where $k_{1}, k_{2}$ and $x_{0}$ are given in part 3 of Theorem 1.3.

Proof. To prove (i), it suffices to show the equation

$$
\lambda_{1} M_{1}=M_{2}^{p}, \quad \lambda_{2} M_{2}=M_{1}^{q} .
$$

This can be easily done by using the system (3.8). Indeed, taking the average of (3.8), one can readily deduce that

$$
\bar{v}_{1}^{\prime}= \pm r^{-1}\left(\lambda_{1} M_{1}-M_{2}^{p}+o(1)\right), \quad \bar{v}_{2}^{\prime}= \pm r^{-1}\left(\lambda_{2} M_{2}-M_{1}^{q}+o(1)\right)
$$

as $r \rightarrow \infty$. In turn, (8.10) must hold.

Now the proof of (ii) and (iii) is essentially the same as that for scalar equations, with Theorem 1.3 playing a crucial role in the present case (one may readily verify all conditions needed in Theorem 1.3). We thus omit it and refer the reader to [19, Lemma 8.2].

Proof of (iii) of Theorem 8.1. By Lemma 8.2, one sees that $\left(\mathbf{H}_{1}\right)$ and $\left(\mathbf{H}_{2}\right)$ in Theorem 1.2 hold, provided

$$
\gamma_{0} \doteq \frac{k_{1}}{\alpha_{1} L_{1}}=\frac{k_{2}}{\alpha_{2} L_{2}},
$$

which can be instantly verified by using the definitions of the structural numbers. Hence Theorem 1.2 immediately implies $\mathbf{u}$ is radially symmetric with respect to the hyperplane

$$
x_{1}=\gamma_{0}\left(x_{0}\right)_{1} .
$$

Clearly the choice of $x_{1}$-direction can be arbitrary, due to the symmetric structure of the system. One concludes the radial symmetry of $\mathbf{u}$ with respect to the point $\gamma_{0} x_{0} \in \mathbf{R}^{n}$ and the proof is complete. 


\section{REFERENCES}

1. A.D. Alexandrov, A characteristic property of the spheres, Ann. Mat. Pura Appl. 58 (1962), 303-315. MR 26:722

2. M.-F. Bidaut-Veron and L. Veron, Nonlinear elliptic equations on compact Riemannian manifolds and asymptotics of Emden equations, Invent. Math., 106 (1991), 489-539; Erratum, Invent. Math. 112 (1993). MR 93a:35045; MR 94b:53069

3. L. Caffarelli, B. Gidas and J. Spruck, Asymptotic symmetry and local behavior of semilinear elliptic equations with critical Sobolev growth, Comm. Pure Appl. Math., 42 (1989), 271-297. MR 90c: 35075

4. R. Courant and D. Hilbert, Methods of Mathematical Physics, Vols. I and II, InterscienceWiley, New York, 1962. MR 16:426a; MR 25:4216

5. D.G. de Figueiredo and P.L. Felmer, A Liouville-type theorem for elliptic systems, Ann. Scuola Norm. Sup. Pisa 21 (1994), no.3, 387-397. MR 95m:35009

6. M. Escobedo and M. Herrero, Boundedness and blow-up for a semilinear reaction-diffusion system, J. Diff. Equation 89 (1991), 176-202. MR 91j:35040

7. M. Escobedo and H. Levine, Critical blow-up and global existence numbers for a weakly coupled system of reaction-diffusion equations, Arch. Rational Mech. Anal. 129 (1995), 47-100. MR 96d:35063

8. B. Gidas, W. Ni and L. Nirenberg, Symmetry of positive solutions of nonlinear ellipttic equations in $\mathbf{R}^{n}$, Math. Anal. Appl., Part A, Academic Press, 1981, pp. 369-402. MR 84a:35083

9. D. Gilbarg and N. Trudinger, Elliptic Partial Differential Equations of Second Order, Springer-Verlag, New York, 1983 (second edition), pp. 369-402. MR 86c:35035

10. H.A. Levine, The role of critical exponents in blow-up theorems, SIAM Review, 3 (1990), 262-298. MR 91j:35135

11. E. Mitidieri, Non-existence of positive solutions of semilinear elliptic systems in $\mathbf{R}^{n}$, Quaderno Matematico, 285 (1992).

12. J. Serrin, A symmetry problem in potential theory, Arch. Rational Mech. Anal. 43 (1971), pp.304-318. MR 48:11545

13. J. Serrin and H. Zou, Non-existence of positive solutions of semilinear elliptic systems, Discourses in Math. Appl., 3, Texas A\&M Univ., College Station, 1994, pp. 55-68. MR 97k:35065

14. J. Serrin and H. Zou, Non-existence of positive solutions of Lane-Emden systems, Differential and Integral Equations, 9 (1996), no.4, 635-653. MR 97f:35056

15. J. Serrin and H. Zou, Existence of positive solutions of the Lane-Emden system, Atti del Sem. Mat. Fis. Univ. Modena 43 (1998), Suppl., 369-380. CMP 99:01

16. L. Simon, Asymptotics for a class of nonlinear evolution equations, with applications to geometric problems, Ann. Math. 118 (1983), 525-571. MR 85b:58121

17. L. Simon, Isolated singularities of extrema of geometric variational problems, Lecture Notes in Math. 1161 (1985), 206-277. MR 87d:58045

18. W. Troy, Symmetry properties in systems of semilinear elliptic equations, J. Differential Equations, 42 (1981), 400-413. MR 83b:35051

19. H. Zou, Symmetry of positive solutions of $\Delta u+u^{p}=0$ in $\mathbf{R}^{n}$, J. Differential Equations 120 (1995), no.1, 46-88. MR 96h:35055

20. H. Zou, Slow decay and the Harnack inequality for positive solutions of $\Delta u+u^{p}=0$ in $\mathbf{R}^{n}$, Differential and Integral Equations 8 (1995), no.6, 1355-1368. MR 96b:35057

21. H. Zou, Symmetry of ground states of semilinear equations with mixed Sobolev growth, Indiana Univ. Math. J. 45 (1996), no.1, 221-240. MR 97h:35051

22. H. Zou, in preparation.

Department of Mathematics, University of Alabama at Birmingham, Birmingham, AlABAMA 35294-0001

E-mail address: zou@math.uab.edu 